Preprint typeset in JHEP style - HYPER VERSION

LPSC 08-190

\title{
Cosmological consequences of Yukawa-unified SUSY with mixed axion/axino cold and warm dark matter
}

\author{
Howard Baer $^{a}$, Markus Haider ${ }^{b, c}$, Sabine $\mathrm{Kraml}^{b}$, Sezen Sekmen ${ }^{d}$ and Heaya Summy ${ }^{a}$ \\ ${ }^{a}$ Dept. of Physics and Astronomy, University of Oklahoma, Norman, OK 73019, USA \\ ${ }^{b}$ Laboratoire de Physique Subatomique et de Cosmologie, UJF Grenoble 1, \\ CNRS/IN2P3, INPG, 53 Avenue des Martyrs, F-38026 Grenoble, France \\ ${ }^{c}$ Inst. of Astro and Particle Physics, University of Innsbruck, A-6020 Innsbruck, Austria \\ ${ }^{d}$ Department of Physics, Middle East Technical Univ., TR-06531 Ankara, Turkey \\ E-mail: baer@nhn.ou.edu, markus.haider@lpsc.in2p3.fr, \\ sabine.kraml@lpsc.in2p3.fr, sezen.sekmen@cern.ch, heaya@nhn.ou.edu
}

ABSTRACT: Supersymmetric models with $t-b-\tau$ Yukawa unification at $M_{G U T}$ qualitatively predict a sparticle mass spectrum including first and second generation scalars at the $3-$ $15 \mathrm{TeV}$ scale, third generation scalars at the (few) $\mathrm{TeV}$ scale and gluinos in the sub-TeV range. The neutralino relic density in these models typically turns out to lie far above the measured dark matter abundance, prompting the suggestion that instead dark matter is composed of an axion/axino mixture. We explore the axion and thermal and non-thermal axino dark matter abundance in Yukawa-unified SUSY models. We find in this scenario that $i$ ). rather large values of Peccei-Quinn symmetry breaking scale $f_{a} \sim 10^{12} \mathrm{GeV}$ are favored and $i$ ). rather large values of GUT scale scalar masses $\sim 10-15 \mathrm{TeV}$ allow for the re-heat temperature $T_{R}$ of the universe to be $T_{R} \gtrsim 10^{6} \mathrm{GeV}$. This allows in turn a solution to the gravitino/Big Bang Nucleosynthesis problem while also allowing for baryogenesis via non-thermal leptogenesis. The large scalar masses for Yukawa-unified models are also favored by data on $b \rightarrow s \gamma$ and $B_{s} \rightarrow \mu^{+} \mu^{-}$decay. Testable consequences from this scenario include a variety of robust LHC signatures, a possible axion detection at axion search experiments, but null results from direct and indirect WIMP search experiments.

KeYwords: Supersymmetry Phenomenology, Supersymmetric Standard Model, Dark Matter. 


\section{Introduction}

The celebrated unification of gauge couplings at scale $M_{G U T} \simeq 2 \times 10^{16} \mathrm{GeV}$ under minimal supersymmetric standard model (MSSM) renormalization group evolution[1] strongly suggests that nature is described by some sort of supersymmetric grand unified theory (SUSY GUT) model at very high energy scales. While the GUT gauge group $S U(5)$ [2] has many compelling and beautiful properties, the gauge group $S O(10)$ has an even greater appeal[3], and also some indirect experimental support in terms of how well see-saw neutrino mass fits into the general scheme四]. While both $S U(5)$ and $S O(10)$ SUSY GUT theories admit gauge coupling unification, $S O(10)$ theories also yield matter unification, in that all superfields of a single Standard Model (SM) generation- plus a SM gauge singlet superfield $\hat{N}^{c}$ containing a right-hand neutrino state- fit neatly into the 16-dimensional spinorial representation $\hat{\psi}_{16}$ of $S O(10)$.

In the simplest $S O(10)$ SUSY GUT models, the MSSM Higgs superfields $-\hat{H}_{u}$ and $\hat{H}_{d^{-}}$ both live in the fundamental representation $\hat{\phi}_{10}$. In these models, the superpotential has the form

$$
\hat{f} \ni f \hat{\psi}_{16} \hat{\psi}_{16} \hat{\phi}_{10}+\cdots
$$

where the dots represent model-dependent terms which, for instance, might include further Higgs fields responsible for the GUT gauge symmetry breaking. The coupling $f$ represents the unified Yukawa coupling of each generation: thus, just as $S U(5)$ models often predict $f_{b}-f_{\tau}$ unification, simple $S O(10)$ SUSY GUT models predict the more restrictive $t-b-\tau$ Yukawa coupling unification at the GUT scale[5].

It is probably fair to say that at this moment no compelling $S O(10)$ SUSY GUT model yet exists. Models based in four spacetime dimensions require large, unwieldy Higgs representations to break the $S O(10)$ GUT symmetry. Newer models formulated in extra spacetime dimensions are able to do away with the large Higgs reps and break the GUT symmetry via compactification of the extra dimensions[6]. In our approach here, we will adopt a pragmatic view, assuming that the MSSM (or MSSM plus gauge singlets) is the correct effective theory describing physics between the weak and GUT scales, and we will explore some of the consequences of requiring the three third generation Yukawa couplings to have a high degree of unification at $M_{G U T}$, as suggested by simple $S O(10)$ SUSY GUT models.

In our calculation to check third generation Yukawa coupling unification, we begin with the measured gauge couplings and third generation fermion masses, and use renormalization group methods to evolve the coupled gauge and Yukawa couplings up to the GUT scale. The calculation ends up being sensitive to the entire SUSY particle mass spectrum through weak scale threshold corrections involved in transitioning between the SM and MSSM effective field theories [7]. The parameter space of the model is given by

$$
m_{16}, m_{10}, M_{D}^{2}, m_{1 / 2}, A_{0}, \tan \beta, \operatorname{sign}(\mu)
$$

where $m_{16}$ is the GUT scale mass of all matter scalars, $m_{10}$ is the GUT scale mass of Higgs scalars, $M_{D}$ is their D-term value, $m_{1 / 2}$ is the (unified) GUT scale gaugino mass, $A_{0}$ is the 
unified GUT scale SSB trilinear term, $\tan \beta \equiv v_{u} / v_{d}$ is the weak scale ratio of Higgs field vevs, and $\mu$ is the superpotential Higgs bilinear term, whose magnitude-but not sign-is determined by the scalar potential minimization conditions.

In practice, the two Higgs field soft breaking terms- $m_{H_{u}}^{2}$ and $m_{H_{d}}^{2}$ - cannot be degenerate at $M_{G U T}$ and still allow for an appropriate radiative breakdown of electroweak symmetry (REWSB). Effectively, $m_{H_{u}}^{2}$ must be less than $m_{H_{d}}^{2}$ at $M_{G U T}$ in order to give $m_{H_{u}}^{2}$ a head start in running towards negative values at $M_{\text {weak }}$. We parametrize the Higgs splitting as $m_{H_{u, d}}^{2}=m_{10}^{2} \mp 2 M_{D}^{2}$ in accord with nomenclature for $D$-term splitting to scalar masses when a gauge symmetry undergoes a breaking which reduces the rank of the gauge group. A $D$-term splitting should apply to matter scalar SSB terms as well; in practice, better Yukawa unification is found when the splitting is only applied to the Higgs SSB terms. Such a GUT scale Higgs mass splitting might arise via GUT scale threshold corrections [8].

In previous work, the above parameter space was scanned over (via random scans [9, 10] and also by more efficient Markov Chain Monte Carlo (MCMC) scans 11]) to search for Yukawa unified solutions using the Isasugra subprogram of IsAJET 12 for sparticle mass computations. The quantity

$$
R=\frac{\max \left(f_{t}, f_{b}, f_{\tau}\right)}{\min \left(f_{t}, f_{b}, f_{\tau}\right)} \quad\left(\text { evaluated at } \mathrm{Q}=\mathrm{M}_{\mathrm{GUT}}\right),
$$

was examined, where solutions with $R \simeq 1$ gave apparent Yukawa coupling unification. For superpotential Higgs mass parameter $\mu>0$ (as favored by $(g-2)_{\mu}$ measurements), Yukawa unified solutions with $R \sim 1$ were found but only for special choices of GUT scale boundary conditions [9, 13, 8, 10, 14, 11]:

$$
m_{16} \sim 3-15 \mathrm{TeV}, A_{0} \sim-2 m_{16}, m_{10} \sim 1.2 m_{16}, m_{1 / 2} \ll m_{16}, \tan \beta \sim 50 .
$$

Models with this sort of boundary conditions were derived even earlier in the context of inverted scalar mass hierarchy models (IMH) which attempt to reconcile suppression of flavor-changing and $C P$-violating processes with naturalness via multi- $\mathrm{TeV}$ first/second generation and sub-TeV scale third generation scalars 15. The Yukawa-unified spectral solutions were thus found in Refs. [10, 11] to occur with the above peculiar choice of boundary conditions as long as $m_{16}$ was in the multi-TeV regime.

Based on the above work 10, 11], the sparticle mass spectra from Yukawa-unified SUSY models are characterized qualitatively by the following conditions:

- first and second generation scalars have masses in the $3-15 \mathrm{TeV}$ regime,

- third generation scalars, $\mu$ and $m_{A}$ have masses in the $\mathrm{TeV}$ to few $\mathrm{TeV}$ regime (owing to the inverted scalar mass hierarchy),

- gauginos, including the gluino, have sub-TeV masses,

- the lightest neutralino $\tilde{\chi}_{1}^{0}$ is nearly pure bino with mass typically $m_{\tilde{\chi}_{1}^{0}} \sim 50-80 \mathrm{GeV}$. 
The presence of a bino-like $\tilde{\chi}_{1}^{0}$ along with multi-TeV scalars gives rise to a neutralino cold dark matter $(\mathrm{CDM})$ relic abundance that is typically in the range $\Omega_{\tilde{\chi}_{1}^{0}} h^{2} \sim 10-10^{4}$, i.e. far above 16] the WMAP measured 17] value

$$
\Omega_{C D M} h^{2}=0.110 \pm 0.006
$$

by several orders of magnitude.

One solution to the CDM problem in Yukawa-unified SUSY models occurs for $m_{16} \sim$ $3 \mathrm{TeV}$, where one can have $m_{\tilde{\chi}_{1}^{0}} \simeq m_{h} / 2$. In this case, the $\tilde{\chi}_{1}^{0}$ would pair-annihilate in the early universe through the light Higgs $h$ resonance at a sufficient rate to obtain the desired relic density [11], and could hence be the stable lightest SUSY particle (LSP). However, since $m_{16}$ is relatively low, Yukawa coupling unification only occurs at the $R \sim 1.09$ level. $^{1}$

Another very compelling way out of the Yukawa-unified dark matter abundance problem occurs if one makes the additional assumption that the strong $C P$ problem is solved by the Peccei-Quinn mechanism[18], which leads to the presence of a light pseudoscalar particle, the axion $a$ [19, 20]. Since we are working in a supersymmetric theory, the axion occurs as part of an axion supermultiplet [21], which contains not only the axion, but a spin0 saxion (with mass of order the weak scale), and a spin- $\frac{1}{2}$ axino $\tilde{a}$. The axino is $R$-parity odd. While the saxion is expected to have a mass of order the SUSY breaking scale, the axino mass is very model dependent, and can lie anywhere in the keV-GeV range 22, 23. The axino then can serve as the LSP instead of the lightest neutralino 24, 25.

In the case of an axino LSP, the supposed neutralino relic abundance is greatly reduced since $\tilde{\chi}_{1}^{0} \rightarrow \tilde{a} \gamma$ decay can occur with a lifetime in the range of $\sim 10^{-5}-10^{1}$ sec (depending on parameters). This decay time is sufficiently short that late-time neutralino decay to axino in the early universe should not upset successful predictions of Big Bang Nucleosynthesis $(\mathrm{BBN})$ 23]. The neutralino abundance then gets reduced by the ratio $m_{\tilde{a}} / m_{\tilde{\chi}_{1}^{0}}$ which can be in the range $10^{-1}-10^{-4}$. The $\tilde{a}$ coming from $\tilde{\chi}_{1}^{0}$ decay would actually constitute warm dark matter as long as $m_{\tilde{a}} \lesssim 1 \mathrm{GeV}[26]$. However, axinos can also be produced thermally in the early universe, and these would consititute CDM as long as $m_{\tilde{a}} \gtrsim 100 \mathrm{keV}[23$, 27]. Thus, in the axino LSP case, we could have a mixed dark matter (DM) scenario 28] with

- thermally produced cold or warm axino DM,

- an admixture of warm axino DM arising from $\tilde{\chi}_{1}^{0} \rightarrow \tilde{a} \gamma$ decays and

- a possibly large presence of cold axion DM.

The axino LSP scenario turns out to be even more compelling cosmologically than just as a means to reconcile the dark matter relic abundance with Yukawa-unified models. In this class of Yukawa-unified solutions with $m_{16}$ in the multi-TeV range, we expect from supergravity theory that scalar SSB terms should be directly related to the gravitino mass $m_{3 / 2}$, and so we also expect the gravitino $\tilde{G}$ to lie in the multi-TeV range. The cosmological gravitino problem- wherein gravitinos produced thermally in the early universe suffer a

\footnotetext{
${ }^{1}$ Another possibility, having $m_{A}$ light enough that $\tilde{\chi}_{1}^{0} \tilde{\chi}_{1}^{0}$ can annihilate through the $A$ resonance, appears to be excluded because these cases violate limits on $B F\left(B_{s} \rightarrow \mu^{+} \mu^{-}\right)$decay 11 .
} 
late-time decay, thus destroying the successful predictions of $\mathrm{BBN}$ - can be avoided. For $m_{3 / 2} \lesssim 5 \mathrm{TeV}$, the re-heat temperature $T_{R}$ must be $T_{R} \lesssim 10^{5} \mathrm{GeV}[29]$, thus creating tension with most viable mechanisms for baryogenesis 30]. However, for $m_{3 / 2} \gtrsim 5 \mathrm{TeV}$, the re-heat bound is much higher: $T_{R} \lesssim 10^{8}-10^{9} \mathrm{GeV}$. This range of $T_{R}$ is too low for thermal leptogenesis (which requires $T_{R} \gtrsim 10^{10} \mathrm{GeV}$ ) [30], but is exactly what is needed for baryogenesis via non-thermal leptogenesis [31], wherein the heavy right-hand neutrino states are not produced thermally, but rather via inflaton decay. It was pointed out in Ref. 32] that this is also the exact range needed to generate a dominantly cold axino DM universe. Thus, the whole scenario fits together to offer a consistent cosmological picture of BBN, non-thermal leptogenesis and CDM composed of axions and/or axinos, and one solves the strong $C P$ problem to boot [32]!

In this paper, we make a detailed study of the cosmological consequences of Yukawaunified SUSY GUT models with an axino LSP. The initial study proposed in Ref. 32] assumed only a negligible component of axion dark matter. In this study, we now fold in the axion contribution to the dark matter abundance. In addition, we have updated the value of $m_{t}$ in our calculations, and refined the Yukawa coupling 1-loop beta function threshold effects in ISAJET. We also incorporate in this study the results of our Markov Chain Monte Carlo (MCMC) approach to finding Yukawa-unified solutions. Whereas the Yukawa-unified solutions found via a random scan in Ref. 32] had $m_{16} \sim 15-20 \mathrm{TeV}$, the more efficient MCMC scans used here are able to find many solutions for $m_{16}$ values as low as $3-10 \mathrm{TeV}$.

The paper is organized as follows. In Sec. 2, we update our sparticle mass predictions for Yukawa-unified SUSY models from ISAJET using 1. an improved beta-function threshold decoupling, 2. an updated value of the top mass $m_{t}=172.6 \mathrm{GeV}$, and 3. Markov Chain Monte Carlo (MCMC) scans of the parameter space. We also exhibit plots of the $b \rightarrow s \gamma$ and $B_{s} \rightarrow \mu^{+} \mu^{-}$branching fractions versus $m_{16}$ and find these favor $m_{16}$ values $\gtrsim 10$ $\mathrm{TeV}$. Moreover, we perform MCMC scans for Yukawa-unified solutions with an alternative spectrum generator, SofTsusy, and compare these results with those gained from IsAJET. In Sec. 3, we review elements of axion and axino dark matter cosmology, including plots of the axion relic abundance, axino relic abundance and neutralino lifetime. In Sec. \&, we discuss the gravitino/BBN problem, non-thermal leptogenesis via inflaton decay, and mixed axion/axino dark matter. Our main findings are located in Sec. 4.3. Here, we calculate all three components of axion/axino dark matter in Yukawa-unified models for $m_{16}$ values of 5, 8, 10 and $15 \mathrm{TeV}$. We explore scenarios wherein axions constitute either most, or hardly any, of the dark matter abundance. Our results are presented in the $m_{\tilde{a}}$ vs. $T_{R}$ plane. We find that models with a rather large value of the Peccei-Quinn breaking scale $f_{a} \sim 10^{12}$ $\mathrm{GeV}$ are favored, as well as models with $m_{16} \sim m_{3 / 2}$ on the high side: $\sim 10-15 \mathrm{TeV}$. In these cases, the re-heat temperature of the universe can range above $10^{6} \mathrm{GeV}$, allowing for a solution to the BBN gravitino problem as well as allowing for non-thermal leptogenesis. Our conclusions are presented in Sec. 5. In an Appendix, we list updated Yukawa-unified benchmark points from IsAJET 7.79. 


\section{Updated spectrum predictions for Yukawa-unified SUSY models}

\subsection{Updated Isajet calculation of sparticle mass spectra}

The ISAJET calculations begin by adopting the fermion mass boundary conditions that $m_{b}^{\overline{D R}}\left(M_{Z}\right)=2.83 \mathrm{GeV}, m_{\tau}^{\overline{D R}}\left(M_{Z}\right)=1.7463 \mathrm{GeV}$, and $m_{t}($ pole $)=172.6 \mathrm{GeV}$, along with the measured gauge couplings; in particular we take $\alpha_{s}^{\overline{M S}}\left(M_{Z}\right)=0.1172$. Note the value of $m_{t}$ represents an update due to recent D0 and CDF measurements [33] over our previous work Ref. 11] which was done using $m_{t}=171 \mathrm{GeV}$. Our results do not change qualitatively upon varying the fermion masses within their error bars 10]. We use the IsAJET 7.79 12] program to perform two loop RG evolution of gauge and Yukawa couplings and all soft SUSY breaking terms. For gauge and Yukawa couplings, IsAJET actually uses an RGE approach wherein the 1-loop beta functions change whenever a SSB threshold is passed over 34, 35]. The Yukawa coupling evolution depends on finite terms from 1-loop MSSM threshold effects [0, 36]; these are implemented at a scale $Q=M_{S U S Y} \equiv \sqrt{m_{\tilde{t}_{L}} m_{\tilde{t}_{R}}}$. The threshold effects cause the entire calculation to depend sensitively on the sparticle mass spectrum, which enters the various loop corrections to $f_{t}, f_{b}$ and $f_{\tau}$. In addition, in the two-loop RG running 37] of SSB terms from $M_{G U T}$ to the weak scale, non-mixing soft terms are frozen out at their own mass scale, while SSB terms that mix are frozen at the scale $M_{S U S Y}[38]$. Complete 1-loop corrections are then applied to all sparticle masses. This approach leads to good agreement with other publicly available sparticle spectra codes 39 when the sparticle masses are all nearby in mass scale. For spectra suffering severe splitting (as will be the case here), the ISAJET multiple decoupling approach attempts to deal with the fact that several mass scales may be present in and around the weak scale 35].

Two updates in the ISAJET 7.79 code affect the running of Yukawa couplings. In early versions of ISAJET, all squark contributions to RG running were decoupled at a common squark mass scale taken to be $m_{\tilde{u}_{L}}$ and all sleptons were decoupled at a common scale taken to be $m_{\tilde{e}_{L}}$. In ISAJET 7.79, the first/second and third generation squarks and sleptons decouple at the values of the corresponding soft SUSY breaking terms: thus, in a case where $m_{16}=10 \mathrm{TeV}$, first/second generation squarks and sleptons decouple around 10 $\mathrm{TeV}$ while third generation squarks and sleptons decouple at a much lower scale around $3 \mathrm{TeV}$. In addition, earlier versions of ISAJET included 2-loop terms for MSSM running between $M_{S U S Y}$ and $M_{G U T}$, but turned these off for $Q<M_{S U S Y}$, where the SM was the expected effective theory. In IsAJET 7.79, the MSSM two-loop terms remain in for

$Q<M_{S U S Y}$, since the scale of decoupling of 2-loop terms is a 3-loop effect. The current version should give a better estimate of Yukawa coupling evolution in models with severe first/second and third generation splitting, as in Yukawa-unified models.

\subsection{Isajet/Softsusy comparison for Yukawa-unified spectra}

Other public spectrum codes follow a different approach than IsAJET and perform a 1step decoupling of SUSY particles at $Q=M_{S U S Y}$. This "all-at-once" transition may lead to some differences in the sparticle masses in particular in the case of a widely split spectrum [38]. It is therefore interesting to compare results from different spectrum codes. 


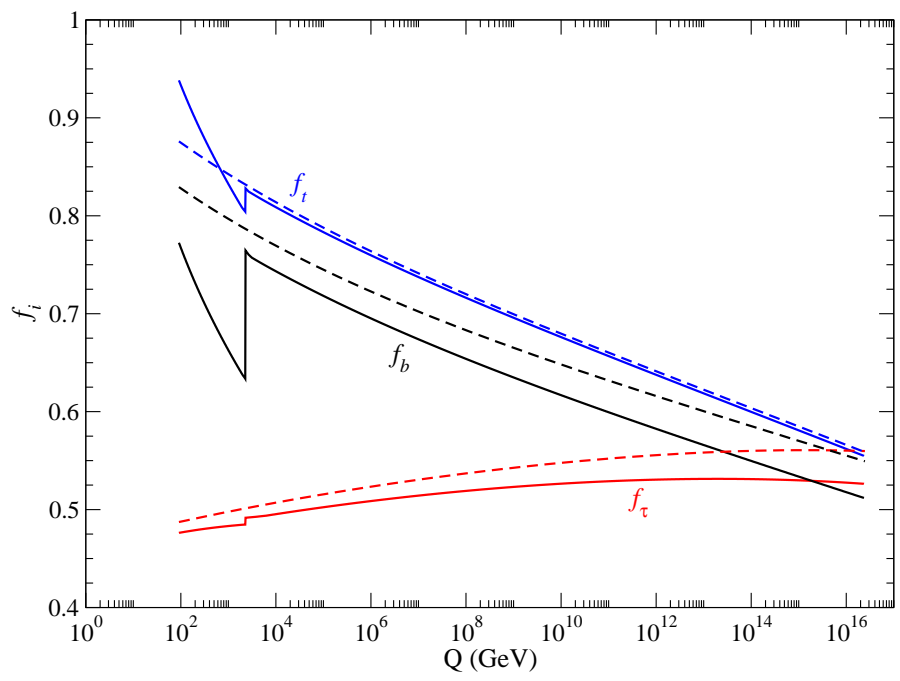

Figure 1: Evolution of $f_{t}, f_{b}$ and $f_{\tau}$ from the weak scale to the GUT scale for the point in Table 1. ISAJET results are solid, while SoftSUsY results are dashed. The large jumps around $3 \mathrm{TeV}$ from ISAJET correspond to the SM-MSSM threshold corrections.

Here, we choose SofTSusy 2.0.18 40] for a representative comparison of the 1-step decoupling with the multi-step approach implemented in ISAJET 7.79.

The evolution of Yukawa couplings between the electroweak and GUT scales is illustrated in Fig. 1 for a point with $m_{16}=8 \mathrm{TeV}$, which runs for both IsAJET and SofTsusy. As can be seen, Softsusy implements threshold corrections to the [gauge and] Yukawa couplings at scale $Q=M_{Z}$, and then runs from $M_{Z}$ to $M_{G U T}$ with full MSSM RG evolution. The SM-MSSM threshold corrections in IsAJET occur at $Q=M_{S U S Y}$, and give rise to the the discontinuities located around $M_{S U S Y} \sim 3 \mathrm{TeV}$. The steep slope for $f_{t}$ and $f_{b}$ for $Q<M_{S U S Y}$ occurs mainly because the co-efficient of the QCD $g_{s}^{2}$ contribution to $f_{t / b}$ running changes from 8 in the SM for $Q<M_{S U S Y}$ to $16 / 3$ in the MSSM for $Q>M_{S U S Y}$. The two programs obviously differ in the bottom Yukawa coupling $f_{b}$. Part of this difference comes from somewhat different weak scale boundary conditions on the value of $m_{b}^{\overline{D R}}\left(M_{Z}\right)$ : as mentioned above, IsAJET has a hard-coded value of $m_{b}^{\overline{D R}}\left(M_{Z}\right)=2.83 \mathrm{GeV}$; in Softsusy, on the other hand, $m_{b}^{\overline{D R}}\left(M_{Z}\right)$ is computed from $m_{b}^{\overline{M S}}\left(m_{b}\right)$ with SUSY corrections added at $M_{Z} \cdot{ }^{2}$ The larger value of $f_{b}$ in SofTsusy causes $f_{\tau}$ to run to slightly higher values at $M_{G U T}$ than for ISAJET . The parameters of the point used and resulting mass spectra are listed in Table 1. Softsusy gives Yukawa unification at 2\%, IsAJET gives Yukawa unification at $8 \%$. While many masses are very similar, the values of $m_{\tilde{g}}$ and especially $m_{A}$ differ quite a bit.

We next scan over the parameter space Eq. (1.2) using a MCMC algorithm, as detailed in [11], that searches for solutions with $R$ as low as possible. Figure 2 shows the degree of Yukawa unification, $R$, found with the two codes as a function of $m_{16}$. Points from IsAJET 7.79 are shown in blue, while points from SofTSUsy 2.0.18 are shown in green. As

\footnotetext{
${ }^{2}$ To comply with the bottom mass used by ISAJET in other parts of the program, we use $m_{b}^{\overline{M S}}\left(m_{b}\right)=$ $4.2 \mathrm{GeV}$ as input in Softsusy, leading to $m_{b}^{\overline{D R}}\left(M_{Z}\right) \simeq 2.6 \mathrm{GeV}$ depending on the exact parameter point.
} 


\begin{tabular}{lcc}
\hline parameter & SOFTSUSY & ISAJET \\
\hline$f_{t}$ & 0.559 & 0.555 \\
$f_{b}$ & 0.549 & 0.512 \\
$f_{\tau}$ & 0.560 & 0.526 \\
$R$ & 1.02 & 1.08 \\
$\mu$ & 2130 & 2178 \\
$m_{\tilde{g}}$ & 332 & 383 \\
$m_{\tilde{u}_{L}}$ & 8004 & 7973 \\
$m_{\tilde{t}_{1}}$ & 1892 & 1812 \\
$m_{\tilde{b}_{1}}$ & 2487 & 2653 \\
$m_{\tilde{e}_{R}}$ & 8064 & 8065 \\
$m_{\tilde{\chi}_{1}^{ \pm}}$ & 116 & 121 \\
$m_{\tilde{\chi}_{2}^{0}}$ & 120 & 120 \\
$m_{\tilde{\chi}_{1}^{0}}$ & 53.4 & 53.1 \\
$m_{A}$ & 681 & 1955 \\
$m_{h}$ & 129.1 & 127.5 \\
\hline
\end{tabular}

Table 1: Masses in GeV units and parameters for a Yukawa-unified point using SofTsusy 2.0.18, IsAJET 7.79. We take $m_{16}=8000 \mathrm{GeV}, m_{10}=9760.3 \mathrm{GeV}, M_{D}=2435.7 \mathrm{GeV}, m_{1 / 2}=67.8752$ $\mathrm{GeV}, A_{0}=-16007 \mathrm{GeV}, \tan \beta=48.71$ and $m_{t}=172.6 \mathrm{GeV}$. Thus, $m_{H_{d}}=10350.3 \mathrm{GeV}$ and $m_{H_{u}}=9132.3 \mathrm{GeV}$

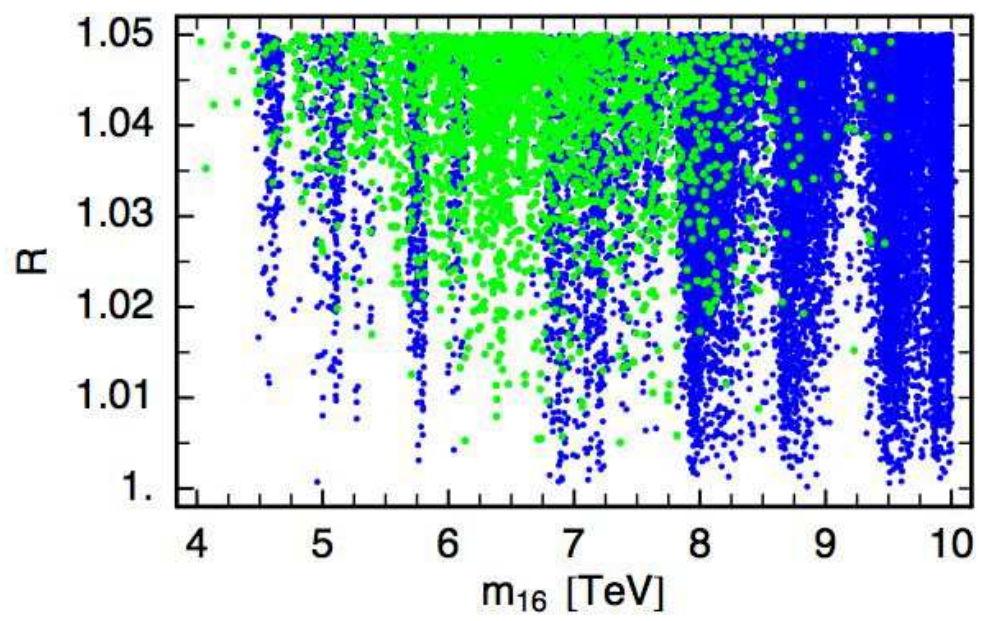

Figure 2: Degree of Yukawa unification, $R$, versus $m_{16}$ from MCMC scans over the $S O(10)$ model parameter space. ISAJET 7.79 results are in blue, while SOFTSUSY 2.0.18 results are in green.

one can see, with both programs a high degree of Yukawa unification can be found, and these solutions tend to prefer high $m_{16}$. However, as is apparent from the density of points, solutions with low $R$ are more easily found with IsAJET than with SofTsusy. Another important difference is that SofTSUSY generates Yukawa-unified solutions only for $m_{16}$ up to about $9.5 \mathrm{TeV}$, while IsAJET generates solutions for $m_{16}$ well beyond $10 \mathrm{TeV}$ (here we only show results up to $10 \mathrm{TeV}$ ). The reason lies in the fine-tuning of the $\mu$ parameter and 

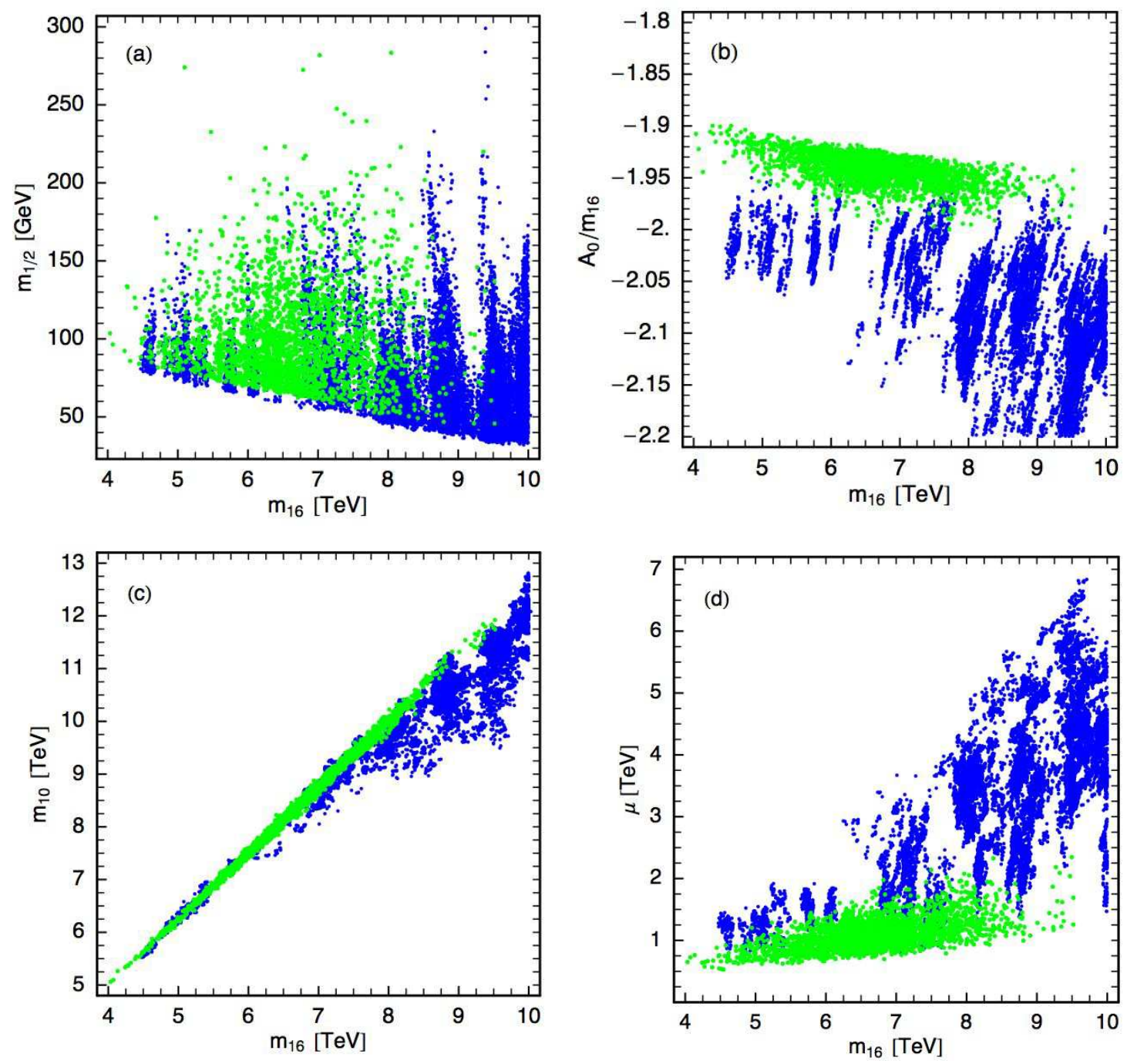

Figure 3: Points with $R \leq 1.05$ from MCMC scans projected into various planes: (a) $m_{1 / 2}$ vs. $m_{16}$, (b) $A_{0} / m_{16}$ vs. $m_{16}$, (c) $m_{10}$ vs. $m_{16}$, and (d) $\mu$ vs. $m_{16}$. IsAJET 7.79 results are in blue, while SOFTSUSY 2.0.18 results are in green.

the electroweak symmetry breaking.

Overall, Yukawa-unified solutions obtained with either program obey the same qualitative conditions Eq. (1.4). This is illustrated in detail in Figs. 3(a)-(d), which show MCMC scan points with $R \leq 1.05$ projected into various planes. In particular, frame (a) shows that $m_{16}$ is in the multi-TeV range while $m_{1 / 2} \sim 100 \mathrm{GeV}$. From frame (b), which shows the $A_{0} / m_{16}$ vs. $m_{16}$ plane, we see that both programs require $A_{0} \sim-2 m_{16}$, although IsAJET prefers somewhat more negative values of $A_{0}$ than Softsusy does. Frame (c) illustrates the correlation $m_{10} \sim 1.2 m_{16}$. Finally, frame (d) shows an important difference in results from the two codes: in spectra obtained with IsAJET the $\mu$ parameter grows with $m_{16}$ and reaches values of a few $\mathrm{TeV}$, while in spectra obtained with Softsusy, the value of $\mu$ 

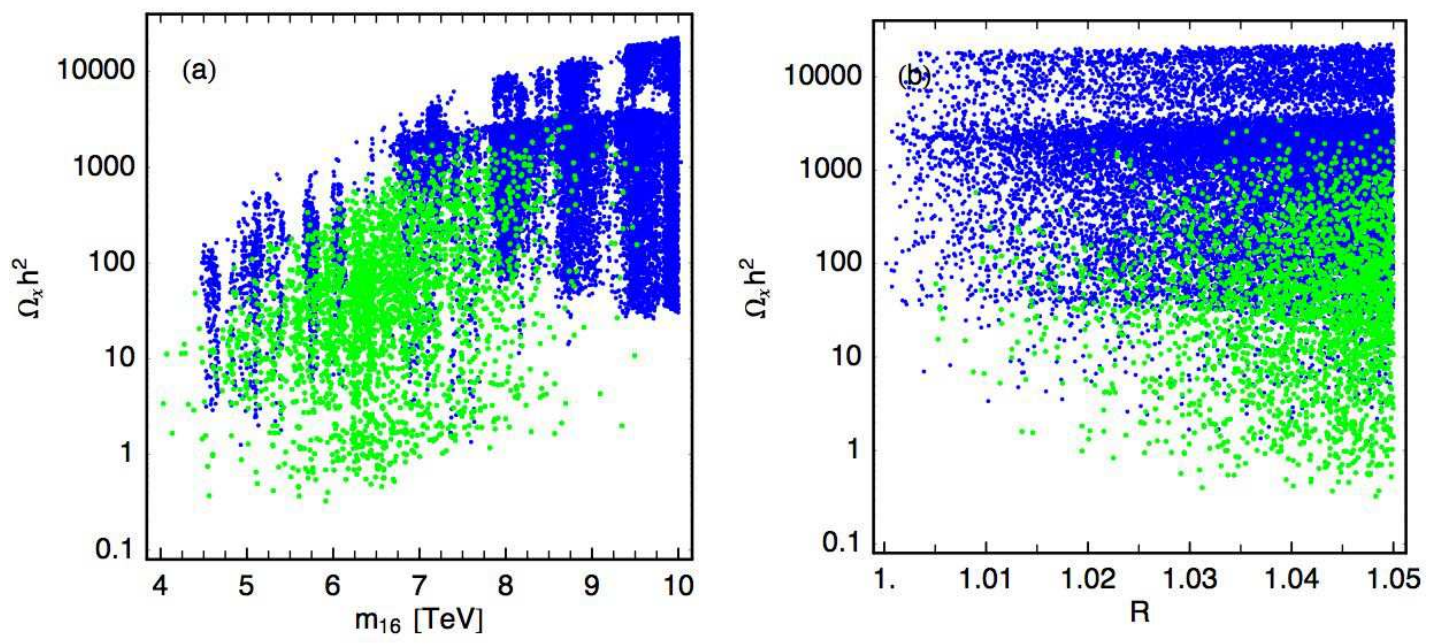

Figure 4: Points with $R \leq 1.05$ from MCMC scans over the $S O(10)$ model parameter space, in (a) $\Omega_{\tilde{\chi}_{1}^{0}} h^{2}$ vs. $m_{16}$ and in (b) $\Omega_{\tilde{\chi}_{1}^{0}} h^{2}$ vs. $R$. IsAJET 7.79 results are in blue, while Softsusy 2.0.18 results are in green.

remains around $1-2 \mathrm{TeV}$.

In Fig. W(a), we show the value of neutralino relic density from both IsAJET and Softsusy versus $m_{16}$ for points with $R \leq 1.05$. The relic density is calculated with Micromegas [41], which easily interfaces with both Isajet and Softsusy. Here, we see SofTSUSY predicts $\Omega_{\tilde{\chi}_{1}^{0}} h^{2} \sim 1-1000$, with some points extending down to $\Omega_{\tilde{\chi}_{1}^{0}} h^{2} \sim 0.3$. These latter points occur due to neutralino annihilation near the light Higgs $h$ resonance, but do not quite reach the WMAP-measured dark matter density because the higgsino fraction $f_{H}$ of the $\tilde{\chi}_{1}^{0}$ is only few per mil. ISAJET tends to give an even smaller higgsino fraction (larger $\mu$ parameter, c.f. Fig. $3($ d) ) and hence an even larger neutralino relic density up to $\Omega_{\tilde{\chi}_{1}^{0}} h^{2} \sim 10000$. Points with $\Omega_{\tilde{\chi}_{1}^{0}} h^{2} \sim 0.1$ due to annihilation through Higgs can be found with both programs, but then the Yukawa unification is only $R \sim 1.07-1.09$ 11 while $f_{H}$ is of the order of $1 \%$. A plot of $\Omega_{\tilde{\chi}_{1}^{0}} h^{2}$ versus $R$ is shown in Fig. 田(b).

We conclude that both ISAJET and SofTSUSY predict a large over-abundance of neutralino dark matter from Yukawa-unified models with $R \leq 1.05$. The postulation of axion/axino dark matter allows one to reconcile the dark matter results with Yukawa unification (as well as solving the strong $C P$ problem).

\subsection{Results for $b \rightarrow s \gamma$ and $B_{s} \rightarrow \mu^{+} \mu^{-}$decay}

In this section, we present results for the branching fractions for $b \rightarrow s \gamma$ and $B_{s} \rightarrow \mu^{+} \mu^{-}$ decays. We adopt the IsAJET Isatools 42 program for these calculations. The $B F(b \rightarrow s \gamma)$ decay rate is calculated in ISAJET [43] by evaluating the Wilson co-efficients for the relevant operators mediating $b \rightarrow s \gamma$ decay at a scale $Q>M_{S U S Y}$, and then running down to $M_{Z}$ scale using a tower of effective theories approach 44. At $M_{Z}$, the Wilson co-efficients are matched to the SM ones, and run using 2-loop evolution down to scale $m_{b}$, where the $b \rightarrow s \gamma$ decay rate is evaluated, including complete NLO corrections 455. Large $\tan \beta$ effects are 


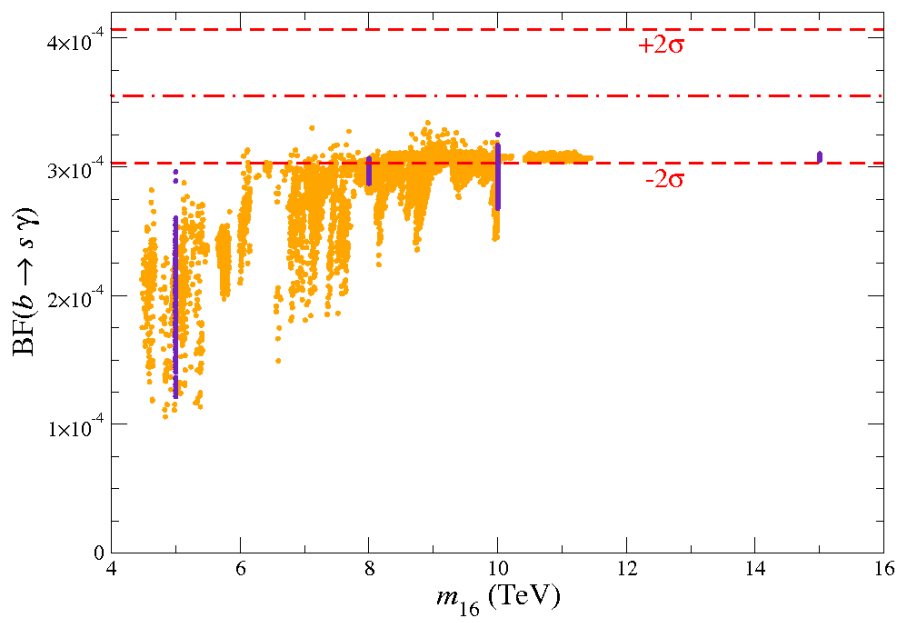

Figure 5: Predictions for $B F(b \rightarrow s \gamma)$ vs. $m_{16}$ from Yukawa-unified models generated by ISAJET 7.79 with $R<1.05$ and $m_{t}=172.6 \mathrm{GeV}$. The blue dots show results from MCMC scans with fixed $m_{16}$. We also show the measured central value of $B F(b \rightarrow s \gamma)$ along with $\pm 2 \sigma$ error bands.

accounted for by adopting the IsAJET running Yukawa couplings, which include threshold effects as noted above 46. The IsAJET SM result, $B F(b \rightarrow s \gamma) \simeq 3.1 \times 10^{-4}$, agrees well with a recent evaluation by Misiak [47], which finds $B F(b \rightarrow s \gamma)_{S M}=(3.15 \pm 0.23) \times 10^{-4}$.

The branching fraction $B F(b \rightarrow s \gamma)$ has been measured by the CLEO, Belle and BABAR collaborations; a combined analysis [48] finds the branching fraction to be $B F(b \rightarrow$

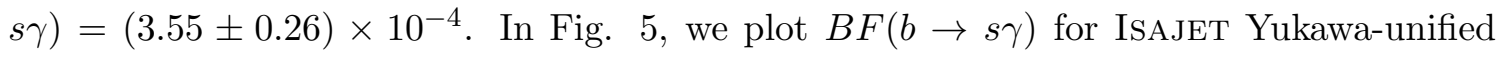
solutions with $R<1.05$ against $m_{16}$. The experimental central value along with $\pm 2 \sigma$ error bands are also shown. The Wilson co-efficients grow with $\tan \beta$, and since we are at $\tan \beta \sim 50$ for Yukawa-unified SUSY, we expect large MSSM contributions to $B F(b \rightarrow s \gamma)$. In fact, we see from Fig. 5 that the $B F(b \rightarrow s \gamma)$ is typically below the measured error bands for $m_{16} \lesssim 10 \mathrm{TeV}$, and as $m_{16}$ gets very large, the branching fraction approaches the SM value. This at least seems to favor $m_{16}$ values $\gtrsim 8-10 \mathrm{TeV}$ in Yukawa-unified models. This observation is also recorded by Altmannshofer et al.[49].

In Fig. [6, we show the branching fraction $B F\left(B_{S} \rightarrow \mu^{+} \mu^{-}\right)$evaluated[50] using Isatools. The experimental 95\% CL upper limit from CDF collaboration is also shown [51]. We see that for lower values of $m_{16}$, in many cases the branching fraction can be near or even above the experimental limit. As $m_{16}$ increases, so does $m_{A}$, which mediates the decay. The large value of $m_{16}$, and hence $m_{A}$, acts to suppress this branching fraction to values about an order of magnitude below present experimental limits.

\section{Axion and axino dark matter}

\subsection{Axion cold dark matter}

The axion arises as a by-product of the Peccei-Quinn solution to the strong $C P$ problem 18 , 


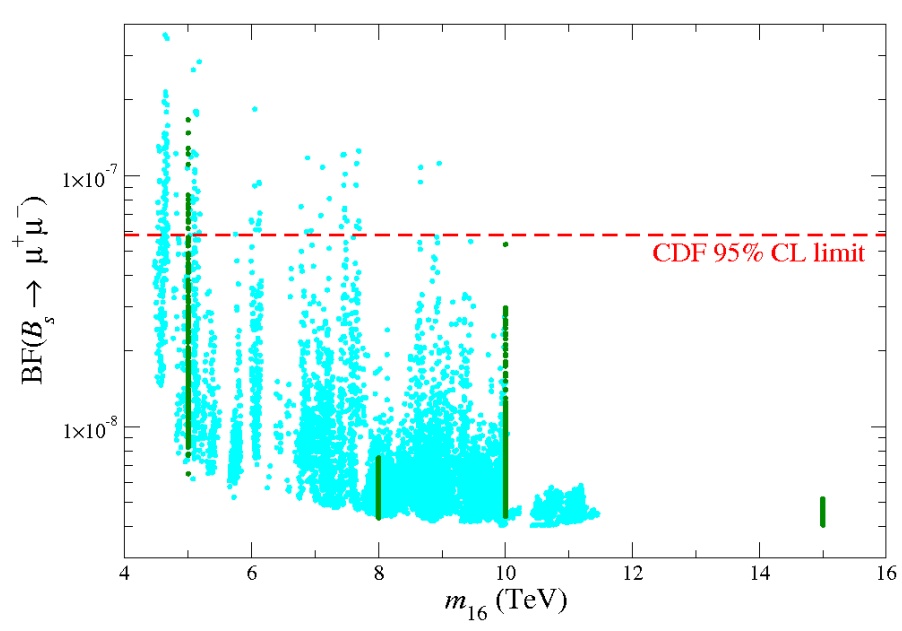

Figure 6: Predictions for $B F\left(B_{s} \rightarrow \mu^{+} \mu^{-}\right)$vs. $m_{16}$ from Yukawa-unified models generated by ISAJET 7.79 with $R<1.05$ and $m_{t}=172.6 \mathrm{GeV}$. The green dots show results from MCMC scans with fixed $m_{16}$. We also show the measured $95 \%$ CL upper limit on this branching fraction from CDF measurements.

20]. The strong $C P$ problem has its origin in an allowed QCD Lagrangian term

$$
\mathcal{L} \ni \frac{\theta g^{2}}{32 \pi^{2}} G_{\mu \nu}^{a} \tilde{G}^{a \mu \nu}
$$

$\left(G_{\mu \nu}^{a}\right.$ is the gluon field strength tensor and $\tilde{G}^{a \mu \nu}$ its dual) which is $P$ and $T$-violating, but $C$ conserving, and hence $C P$ violating. When QCD is coupled to the electroweak theory, $\theta$ is replaced by $\bar{\theta} \equiv \theta+\arg \left(\operatorname{det} m_{q}\right)$, where $m_{q}$ is the quark mass matrix. The measured value of the neutron electric dipole moment (EDM) requires $\bar{\theta} \lesssim 10^{-10}$. Explaining the tininess of this Lagrangian term is the strong $C P$ problem.

The Peccei-Quinn solution to the strong $C P$ problem invokes a theory with a global $U(1)$ (Peccei-Quinn or PQ) symmetry, which is classically valid, but broken spontaneously, and by quantum anomalies. A consequence of the broken PQ symmetry is the existence of a pseudo-Goldstone boson field: the axion $a(x)$ [19]. In this case, the Lagrangian also contains the terms

$$
\mathcal{L} \ni \frac{1}{2} \partial_{\mu} a \partial^{\mu} a+\frac{g^{2}}{32 \pi^{2}} \frac{a(x)}{f_{a} / N} G_{\mu \nu}^{a} \tilde{G}^{a \mu \nu}
$$

where we have introduced the PQ breaking scale $f_{a}$ and $N$ is the model-dependent color anomaly of order 1 . The effective potential for the axion field $V(a(x))$ has its minimum at $\langle a(x)\rangle=-\bar{\theta} f_{a} / N$, and so the offending $G \tilde{G}$ term essentially vanishes, which solves the strong $C P$ problem. A consequence of this very elegant mechanism is that a physical axion field should exist, with concommitant particle excitations.

The axion mass can be computed using current algebra techniques, and is given by

$$
m_{a} \simeq 6 \mathrm{eV} \frac{10^{6} \mathrm{GeV}}{f_{a} / N}
$$

The axion field couples to gluon-gluon (obvious from Eq. (3.2) ) and also to photon-photon and fermion-fermion. All the couplings are suppressed by the PQ scale $f_{a}$. 
Astrophysical limits from cooling of red giant stars and supernova 1987a require $f_{a} / N \gtrsim 10^{9} \mathrm{GeV}$, or $m_{a} \lesssim 3 \times 10^{-3} \mathrm{eV}$. In addition, axions can be produced via various mechanisms in the early universe. Since their lifetime (they decay via $a \rightarrow \gamma \gamma$ ) turns out to be longer than the age of the universe, they can be a good candidate for dark matter in the universe. Since we will be concerned here with re-heat temperatures of the universe $T_{R} \lesssim 10^{9} \mathrm{GeV}<f_{a}$ (to avoid overproducing gravitinos in the early universe), the axion production mechanism relevant for us here is just one: production via vacuum mis-alignment [52]. In this mechanism, the axion field $a(x)$ can have any value $\sim f_{a}$ at temperatures $T \gg \Lambda_{Q C D}$. As the temperature of the universe drops, the potential turns on, and the axion field oscillates and settles to its minimum at $-\bar{\theta} f_{a} / N$. The difference in axion field before and after potential turn-on corresponds to the vacuum mis-alignment: it produces an axion number density

$$
n_{a}(t) \sim \frac{1}{2} m_{a}(t)\left\langle a^{2}(t)\right\rangle,
$$

where $t$ is the time near the QCD phase transition. Relating the number density to the entropy density allows one to determine the axion relic density today:

$$
\Omega_{a} h^{2} \simeq \frac{1}{4}\left(\frac{6 \times 10^{-6} \mathrm{eV}}{m_{a}}\right)^{7 / 6} .
$$

An error estimate of the axion relic density from vacuum mis-alignment is plus-or-minus a factor of three. Axions produced via vacuum mis-alignment would constititute cold dark matter.

The axion relic density from vacuum mis-alignment, along with error bands, is shown in Fig. 1. However, in the event that $\left\langle a^{2}(t)\right\rangle$ is inadvertently small, then much lower values of relic density could be allowed (or much higher if $\left\langle a^{2}(t)\right\rangle$ is inadvertently large). Additional entropy production at $t>t_{Q C D}$ can also lower the axion relic abundance. Taking the value of Eq. (3.5) literally, and comparing to the WMAP5 measured abundance of CDM in the universe, one gets an upper bound $f_{a} / N \lesssim 5 \times 10^{11} \mathrm{GeV}$, or a lower bound $m_{a} \gtrsim 10^{-5}$ $\mathrm{eV}$. If we take the axion relic density a factor of three lower, then the bounds change to $f_{a} / N \lesssim 1.2 \times 10^{12} \mathrm{GeV}$, and $m_{a} \gtrsim 4 \times 10^{-6} \mathrm{eV}$.

\subsection{Warm and cold axino dark matter}

\subsubsection{Non-thermally produced axino dark matter}

Since we are working in a supersymmetric model, the axion field will be only one element of an axion left chiral scalar superfield

$$
\hat{\phi}_{a}=\frac{s(\hat{x})+i a(\hat{x})}{\sqrt{2}}+i \sqrt{2} \bar{\theta} \psi_{a_{L}}(\hat{x})+i \bar{\theta} \theta_{L} \mathcal{F}_{a}(\hat{x})
$$

where $\theta$ here are the anti-commuting Grassman superspace dimensions arranged in a Majorana spinor, and $\hat{x}_{\mu}=x_{\mu}+\frac{i}{2} \bar{\theta} \gamma_{5} \gamma_{\mu} \theta[53]$. The superfield $\hat{\phi}_{a}$ contains the $R$-even spin- 0 saxion field $s$, which gets a mass of order the SUSY breaking scale, and the $R$-odd spin- $\frac{1}{2}$ 


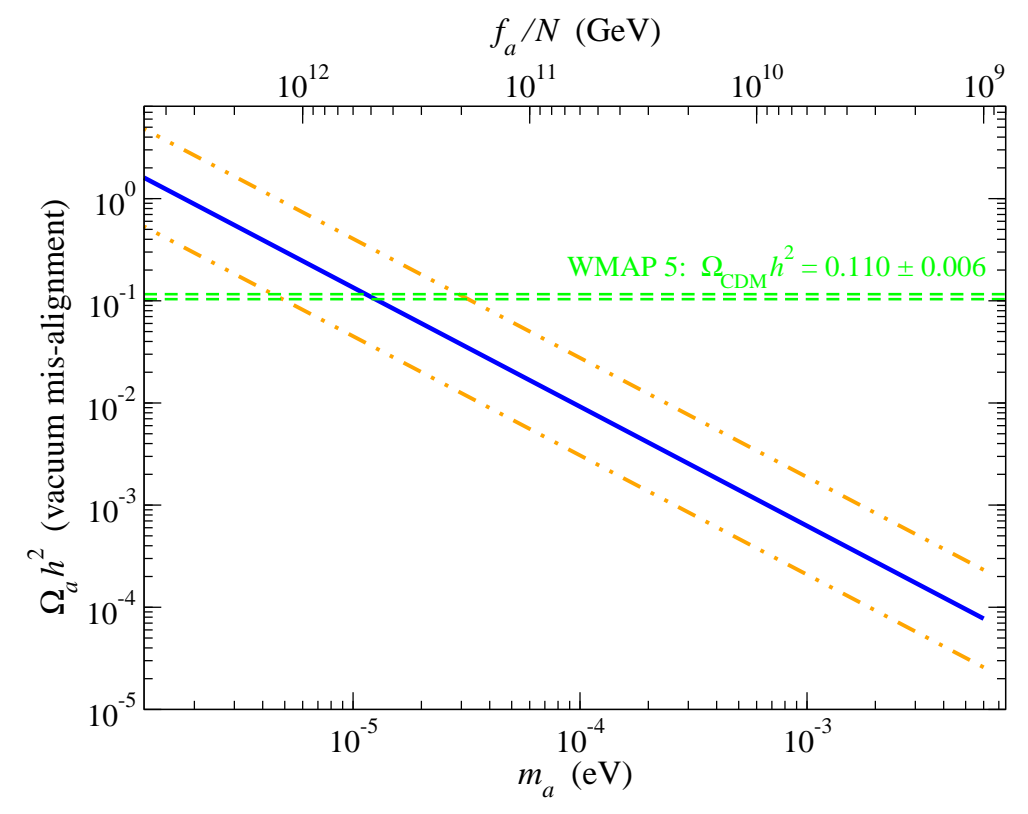

Figure 7: Axion relic density due to vacuum mis-alignment versus $m_{a}$ (lower scale) and $f_{a} / N$ (upper scale). The plot includes a factor three error estimate along with the WMAP5 CDM measured abundance.

axino field $\psi_{a} \equiv \tilde{a}$, whose mass is model-dependent, and can range over the keV-GeV scale[22, 23].

Here, we assume that the $\tilde{a}$ is the LSP, so that the neutralino is in fact unstable, and decays dominantly into $\tilde{\chi}_{1}^{0} \rightarrow \tilde{a} \gamma$. The width $\Gamma\left(\tilde{\chi}_{1}^{0} \rightarrow \tilde{a} \gamma\right)$ has been calculated in Ref. [23], and is given by

$$
\Gamma\left(\tilde{\chi}_{1}^{0} \rightarrow \tilde{a} \gamma\right)=\frac{\alpha_{e m}^{2} C_{a Y Y} v_{4}^{(1) 2}}{128 \pi^{3} \cos ^{2} \theta_{W}} \frac{m_{\tilde{\chi}_{1}^{0}}^{3}}{\left(f_{a} / N\right)^{2}}\left(1-\frac{m_{\tilde{a}}^{2}}{m_{\tilde{\chi}_{1}^{0}}^{2}}\right)^{3}
$$

where $v_{4}^{(1)}$ denotes the bino fraction of neutralino $\tilde{\chi}_{1}^{0}, N$ is the model-dependent anomaly factor (e.g. $N=1$ (6) for KSVZ[54 (DFSZ [55]) axions), and $C_{a Y Y}$ is a model-dependent coupling factor (e.g. $C_{a Y Y}=8 / 3$ in the DFSZ model).

In Fig. 8, we plot the $\tilde{\chi}_{1}^{0}$ lifetime in seconds versus $m_{\tilde{\chi}_{1}^{0}}$ for four choices of $f_{a} / N$, and taking $C_{a Y Y}=8 / 3$. The lifetime ranges from $\sim 10^{-5}$ s for $f_{a} / N=10^{9} \mathrm{GeV}$, up to $\sim 40$ s for $f_{a} / N=10^{12} \mathrm{GeV}$. In the latter case, the $\tilde{\chi}_{1}^{0}$ will decay while BBN is ongoing. The dominant decay into a high energy photon should thermalize with the electron-nucleon plasma. It is of note that $\tilde{\chi}_{1}^{0} \rightarrow q \bar{q} \tilde{a}$ three body hadronic decays via intermediate $\gamma$ and $Z$ can also occur at a small branching fraction. These hadronic decays would be more likely to be a threat to disrupt Big Bang Nucleosynthesis. Also, $\tilde{\chi}_{1}^{0} \rightarrow Z \tilde{a}$ can occur, but only for $m_{\tilde{\chi}_{1}^{0}}>M_{Z}$, which rarely occurs in our Yukawa-unified scenario.

The axino dark matter produced from neutralino decay would compose non-thermally produced (NTP) dark matter. Jedamzik et al. 26] have calculated the rms velocity profile of axino dark matter coming from neutralino decay. A comparison against data from Lyman 


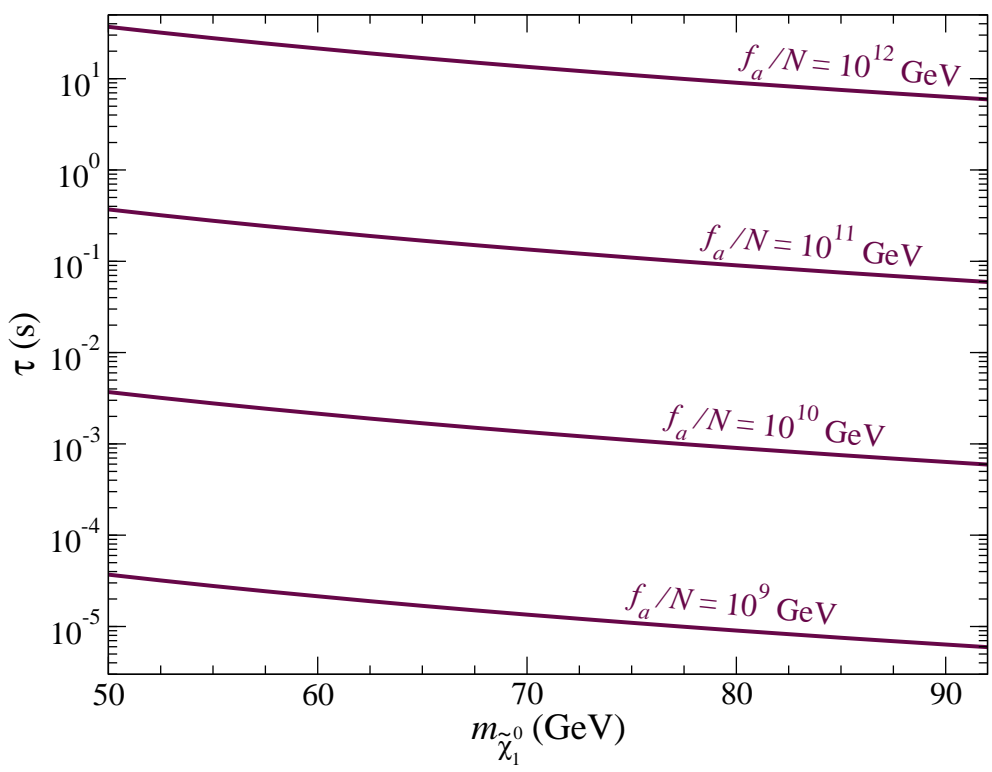

Figure 8: Lifetime in seconds of the lightest neutralino versus its mass for $f_{a} / N=10^{9}, 10^{10}, 10^{11}$ and $10^{12} \mathrm{GeV}$, respectively.

alpha forest leads them to conclude that non-thermally produced axinos will consitute warm dark matter for $m_{\tilde{a}} \lesssim 1 \mathrm{GeV}$.

The relic abundance of non-thermally produced axinos can be simply obtained from the neutralino abundance. The neutralino thermal abundance calculation proceeds by solving the Boltzmann equation for neutralinos from freeze-out to the present day, after inputting the usual neutralino annihilation and co-annihilation cross sections. Since each neutralino decays to one axino, the axinos inherit the neutralino number density, and the non-thermally produced axino abundance is simply

$$
\Omega_{\tilde{a}}^{\mathrm{NTP}} h^{2}=\frac{m_{\tilde{a}}}{m_{\tilde{\chi}_{1}^{0}}} \Omega_{\tilde{\chi}_{1}^{0}} h^{2} .
$$

In this regard, if the ratio $m_{\tilde{a}} / m_{\tilde{\chi}_{1}^{0}}$ is small, then large factors of neutralino dark matter density can be shed by undergoing $\tilde{\chi}_{1}^{0} \rightarrow \tilde{a} \gamma$ decay. It is this mechanism that allows one to reconcile the huge neutralino relic abundance from Yukawa-unified models with the WMAP measured abundance.

\subsubsection{Thermally produced axino dark matter}

In our scenario, where we only consider $T_{R} \ll f_{a}$, the axinos in the early universe are too weakly interacting to be in thermal equilibrium. Nevertheless, they can be produced by radiation off other particles which are in the thermal bath, much the same as gravitinos can be produced in the early universe. Initial calculations of the thermally produced (TP) axino abundance were performed in Ref. [23], wherein a variety of QCD axino production processes (such as $g g \rightarrow \tilde{a} \tilde{g}, g \tilde{q} \rightarrow \tilde{a} q, \cdots$ ) were considered. Divergent diagrams involving $t$-channel exchange of massless gluons were regulated by introducing a "plasmon" mass, representing the effective gluon mass in the plasma of the early universe. A later evaluation 


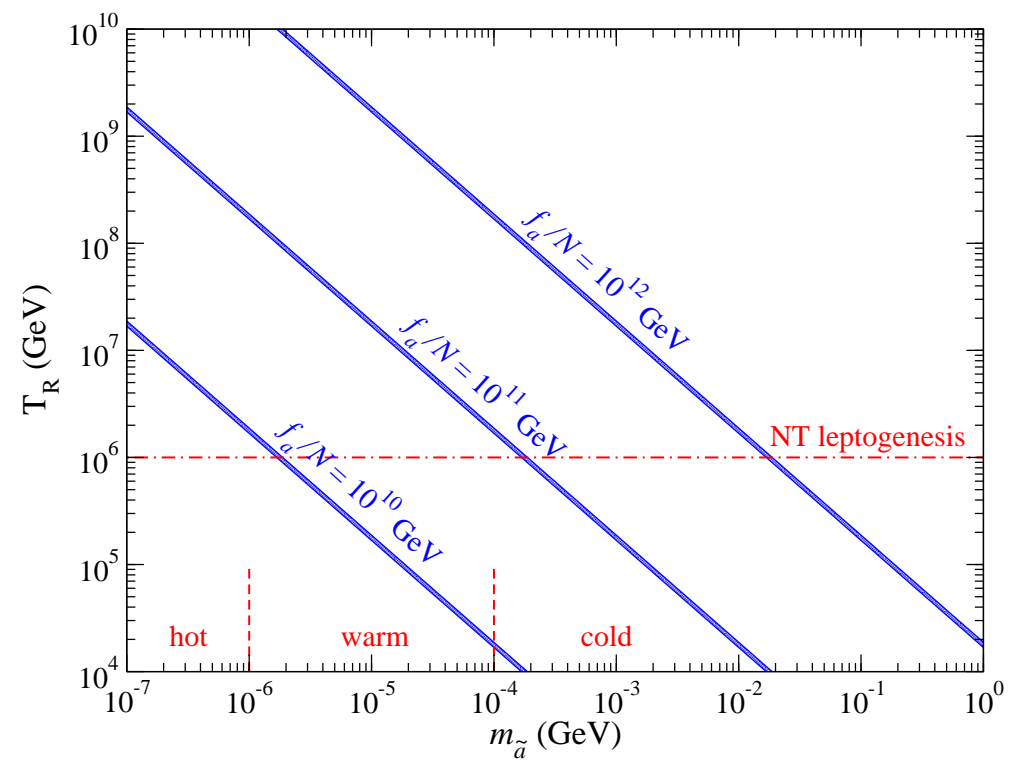

Figure 9: Thermally produced axino relic density within the WMAP5 measured limits in the $m_{\tilde{a}}$ vs. $T_{R}$ plane for $f_{a} / N=10^{10}, 10^{11}$ and $10^{12} \mathrm{GeV}$, respectively.

of thermally produced axino matter in Ref. 27] used the hard thermal loop resummation technique of Braaten-Pisarski[56] and obtained a reduced axino dark matter yield by a factor of $\sim 3$. The results of Ref. [27] are summarized in the expression

$$
\Omega_{\tilde{a}}^{\mathrm{TP}} h^{2} \simeq 5.5 g_{s}^{6} \ln \left(\frac{1.108}{g_{s}}\right)\left(\frac{10^{11} \mathrm{GeV}}{f_{a} / N}\right)^{2}\left(\frac{m_{\tilde{a}}}{0.1 \mathrm{GeV}}\right)\left(\frac{T_{R}}{10^{4} \mathrm{GeV}}\right)
$$

where $g_{s}$ is the strong coupling evaluated at $Q=T_{R}$ (e.g. $g_{s}=.915$ at $Q=10^{6} \mathrm{GeV}$ from our IsAJET RGE calculations). The thermally produced axinos qualify as cold dark matter as long as $m_{\tilde{a}} \gtrsim 100 \mathrm{keV} 23,27$.

In Fig. 9, we show bands of the $m_{\tilde{a}} v s . T_{R}$ plane which give $\Omega_{\tilde{a}}^{\mathrm{TP}} h^{2}$ within the WMAPmeasured dark matter abundance, for $f_{a} / N=10^{10}, 10^{11}$ and $10^{12} \mathrm{GeV}$. We see that for the lower range of $f_{a} / N \sim 10^{10} \mathrm{GeV}$, very low values of $m_{\tilde{a}}$ and $T_{R}$ are required. In this case, with $m_{\tilde{a}} \lesssim 100 \mathrm{keV}$, the thermally produced axino DM would likely constitute warm $\mathrm{DM}$, and furthermore, the low value of $T_{R}$ excludes some of the possible mechanisms for baryogenesis. In this case, if we want $T_{R} \gtrsim 10^{6} \mathrm{GeV}$ and $m_{\tilde{a}} \gtrsim 100 \mathrm{keV}$ with dominant thermal production of cold axino DM, then we will need higher $f_{a} / N \gtrsim 10^{11} \mathrm{GeV}$.

In Fig. 10, we show bands of $\Omega_{\tilde{a}}^{\mathrm{TP}} h^{2}=0.11,0.03,0.01$ and 0.001 in the $m_{\tilde{a}}$ vs. $T_{R}$ plane for $f_{a} / N=10^{12} \mathrm{GeV}$. In this case, if the thermally produced axino dark matter only constitutes a small fraction of the total dark matter, and most of the remainder is composed of cold axions, then much smaller values of $m_{\tilde{a}}$ are allowed, and the thermally produced axinos can be either warm or even hot dark matter. 


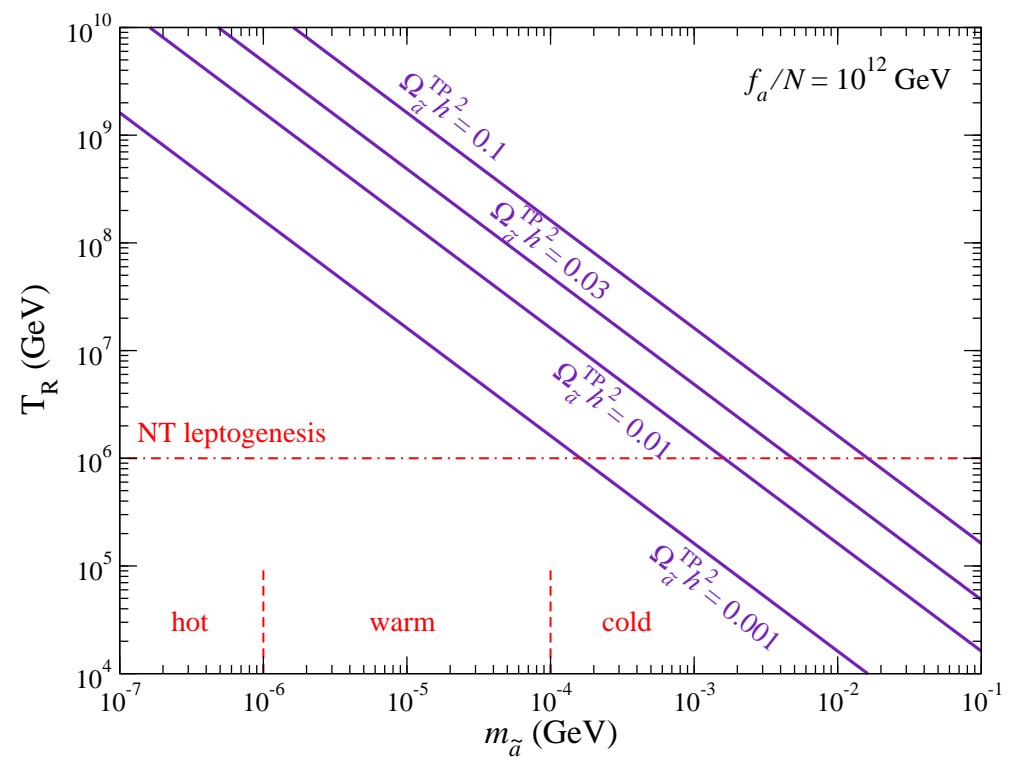

Figure 10: Thermally produced axino relic density in the $m_{\tilde{a}}$ vs. $T_{R}$ plane for $f_{a} / N=10^{12} \mathrm{GeV}$.

\section{The gravitino problem, non-thermal leptogenesis and mixed axion/axino dark matter}

\subsection{The gravitino problem}

A problem common to all SUSY models including supergravity (SUGRA) is known as the gravitino problem. In realistic SUGRA models (those that include the SM as their sub-weak-scale effective theory), SUGRA is broken in a hidden sector by the superHiggs mechanism. A mass for the gravitino $\tilde{G}$ is induced by SUGRA breaking, which is commonly taken to be of order the weak scale. The gravitino mass $m_{3 / 2}$ sets the mass scale for all the soft breaking terms, so that all SSB terms end up also being of order the weak scale [57, 58].

The coupling of the gravitino to matter is strongly suppressed by the Planck mass, so the $\tilde{G}$ in the mass range considered here $\left(m_{3 / 2} \sim m_{16} \sim 5-20 \mathrm{TeV}\right)$ is never in thermal equilibrium with the thermal bath in the early universe. Nonetheless, it does get produced by scatterings of particles that do partake of thermal equilibrium. Thermal production of gravitinos in the early universe has been calculated in Refs. [59], where the abundance is found to depend naturally on $m_{3 / 2}$ and on the re-heat temperature $T_{R}$ at the end of inflation. Once produced, the $\tilde{G}$ s decay into all varieties of particle-sparticle pairs, but with a lifetime that can exceed $\sim 1$ sec, the time scale where Big Bang nucleosynthesis (BBN) begins. The energy injection from $\tilde{G}$ decays is a threat to dis-associate the light element nuclei which are created in BBN. Thus, the long-lived $\tilde{G}$ s can destroy the successful predictions of the light element abundances as calculated by nuclear thermodynamics.

The BBN constraints on gravitino production in the early universe have been calculated by several groups 60]. The recent results from Ref. [29] give an upper limit on the reheat temperature as a function of $m_{3 / 2}$. The results depend on how long-lived the $\tilde{G}$ is (at what stage of BBN the energy is injected), and what its dominant decay modes are. 
Qualitatively, for $m_{3 / 2} \lesssim 5 \mathrm{TeV}, T_{R} \lesssim 10^{6} \mathrm{GeV}$ is required; if this is violated, then too many $\tilde{G}$ are produced in the early universe, which detroy the ${ }^{3} \mathrm{He},{ }^{6} \mathrm{Li}$ and $\mathrm{D}$ abundance calculations. For $m_{3 / 2} \sim 5-50 \mathrm{TeV}$, the re-heat upper bound is much less: $T_{R} \lesssim 10^{9}$ $\mathrm{GeV}$ (depending on the ${ }^{4} \mathrm{He}$ abundance) due to overproduction of ${ }^{4} \mathrm{He}$ arising from $n \leftrightarrow p$

conversions. For $m_{3 / 2} \gtrsim 50 \mathrm{TeV}$, there is an upper bound of $T_{R} \lesssim 10^{10} \mathrm{GeV}$ due to overproduction of $\tilde{\chi}_{1}^{0}$ LSPs due to $\tilde{G}$ decays.

Solutions to the gravitino BBN problem then include: 1 . having $m_{3 / 2} \gtrsim 50 \mathrm{TeV}$ but with an unstable $\tilde{\chi}_{1}^{0}$ ( $R$-parity violation and no $T_{R}$ bound), 2 . having a gravitino LSP so that $\tilde{G}$ is stable or 3 . keep the re-heat temperature below the BBN bounds. We will here adopt solution number 3 . In the case of $S O(10)$ SUSY GUT models, we expect $m_{3 / 2} \sim m_{16}$ and since $m_{16} \sim 5-20 \mathrm{TeV}$, this means we need a re-heat temperature $T_{R} \lesssim 10^{9} \mathrm{GeV}$.

\subsection{Non-thermal leptogenesis}

The data gleaned on neutrino masses during the past decade has led credence to a particular mechanism of generating the baryon asymmetry of the universe known as leptogenesis 61. Leptogenesis requires the presence of heavy right-handed gauge-singlet Majorana neutrino states $\psi_{N_{i}^{c}}\left(\equiv N_{i}\right)$ with mass $M_{N_{i}}$ (where $i=1-3$ is a generation index). The $N_{i}$ states may be produced thermally in the early universe, or perhaps non-thermally, as suggested in Ref. 62] via inflaton $\phi \rightarrow N_{i} N_{i}$ decay. The $N_{i}$ may then decay asymmetrically to elements of the doublets- for instance $\Gamma\left(N_{1} \rightarrow h_{u}^{+} e^{-}\right) \neq \Gamma\left(N_{1} \rightarrow h_{u}^{-} e^{+}\right)$- owing to the contribution of $C P$ violating phases in the tree/loop decay interference terms. Focusing on just one species of heavy neutrino $N_{1}$, the asymmetry is calculated to be 63

$$
\epsilon \equiv \frac{\Gamma\left(N_{1} \rightarrow \ell^{+}\right)-\Gamma\left(N_{1} \rightarrow \ell^{-}\right)}{\Gamma_{N_{1}}} \simeq-\frac{3}{8 \pi} \frac{M_{N_{1}}}{v_{u}^{2}} m_{\nu_{3}} \delta_{\mathrm{eff}}
$$

where $m_{\nu_{3}}$ is the heaviest active neutrino, $v_{u}$ is the up-Higgs vev and $\delta_{\text {eff }}$ is an effective $C P$-violating phase factor which may be of order 1 . The ultimate baryon asymmetry of the universe is proportional to $\epsilon$, so larger values of $M_{N_{1}}$ lead to a higher baryon asymmetry.

To find the baryon asymmetry, one may first assume that the $N_{1}$ is thermally produced in the early universe, and then solve the Boltzmann equations for the $B-L$ asymmetry. The ultimate baryon asymmetry of the universe arises from the lepton asymmetry via sphaleron effects. The final answer 64, compared against the WMAP-measured result $\frac{n_{B}}{s} \simeq 0.9 \times 10^{-10}$ for the baryon-to-entropy ratio, requires $M_{N_{1}} \gtrsim 10^{10} \mathrm{GeV}$, and thus a re-heat temperature $T_{R} \gtrsim 10^{10} \mathrm{GeV}$. This high a value of reheat temperature is in conflict with the upper bound on $T_{R}$ discussed in Sec. 4.1. In this way, it is found that generic SUGRA models with $R$-parity conservation are apparently in conflict with thermal leptogenesis as a means to generate the baryon asymmetry of the universe.

If one instead looks to non-thermal (NT) leptogenesis, then it is possible to have lower reheat temperatures, since the $N_{1}$ may be generated via inflaton decay. The Boltzmann equations for the $B-L$ asymmetry have been solved numerically in Ref. [65]. The $B-L$ asymmetry is then converted to a baryon asymmetry via sphaleron effects as usual. The 
baryon-to-entropy ratio is found to be 65

$$
\frac{n_{B}}{s} \simeq 8.2 \times 10^{-11} \times\left(\frac{T_{R}}{10^{6} \mathrm{GeV}}\right)\left(\frac{2 M_{N_{1}}}{m_{\phi}}\right)\left(\frac{m_{\nu_{3}}}{0.05 \mathrm{eV}}\right) \delta_{e f f},
$$

where $m_{\phi}$ is the inflaton mass. Comparing calculation with data, a lower bound $T_{R} \gtrsim 10^{6}$ $\mathrm{GeV}$ may be inferred for viable non-thermal leptogenesis via inflaton decay.

\subsection{A consistent cosmology for Yukawa-unified models with mixed axion/axino dark matter}

Next, we investigate various scenarios with mixed axion/axino cold and warm dark matter, first to see if they can yield a consistent cosmology, and second, to see if cosmology provides any insight into allowed model parameters. Here, we will investigate four cases.

1. We take $f_{a} / N=10^{11} \mathrm{GeV}$. Using the central value from Eq. 3.5, we obtain a small fraction of axion CDM: $\Omega_{a} h^{2} \simeq 0.017$. The bulk of CDM must then be composed of something else: in our case, thermally produced axinos (so $m_{\tilde{a}} \gtrsim 100 \mathrm{keV}$ ). We take $\Omega_{\tilde{a}}^{\mathrm{TP}} h^{2}=0.083$. Then to obtain the WMAP5 measured DM abundance, we get $\Omega_{\tilde{a}}^{\mathrm{NTP}} h^{2} \sim 0.01$.

2. We take $f_{a} / N=4 \times 10^{11} \mathrm{GeV}$ so that the central value of axion relic abundance Eq. 3.5 yields $\Omega_{a} h^{2} \simeq 0.084$. This gives dominant axion CDM, so that thermally and non-thermally produced axino abundance should be small. Here we assume an equal mix of thermal and non-thermal axinos, so $\Omega_{\tilde{a}}^{\mathrm{TP}} h^{2}=\Omega_{\tilde{a}}^{\mathrm{NTP}} h^{2}=0.013$.

3. We take $f_{a} / N=10^{12} \mathrm{GeV}$, and a factor of $1 / 3$ error on $\Omega_{a} h^{2}$ as in the lower dashed curve of Fig. 7. Thus, $\Omega_{a} h^{2}=0.084$ so we have dominant axion CDM. As in the previous case we take an equal mix of thermally and non-thermally produced axinos: $\Omega_{\tilde{a}}^{T P} h^{2}=\Omega_{\tilde{a}}^{N T P} h^{2}=0.013$.

4. Here, we again take $f_{a} / N=10^{12} \mathrm{GeV}$, but assume the axion vev is accidentally close to zero so that it is nearly aligned with the potential minimum, instead of mis-aligned. Even though $f_{a} / N$ is large, the resulting axion abundance is small: $\Omega_{a} h^{2} \sim 0$. In this case, thermally produced axinos should make up the dominant CDM component. We take $\Omega_{\tilde{a}}^{\mathrm{TP}} h^{2}=0.1$ and $\Omega_{\tilde{a}}^{\mathrm{NTP}} h^{2} \sim 0.01$. This case was shown previously as one adopted in Ref. [32].

Once the value of $\Omega_{\tilde{a}}^{\mathrm{NTP}} h^{2}$ is known, we may calculate $\Omega_{\tilde{\chi}_{1}^{0}} h^{2}$ and $m_{\tilde{\chi}_{1}^{0}}$ in any supersymmetric model (with a $\tilde{\chi}_{1}^{0} \mathrm{NLSP}$ ) and use Eq. (3.8) to calculate the value of $m_{\tilde{a}}$ that is needed. Then, if a value of $f_{a} / N$ has been selected, and we know $\Omega_{\tilde{a}}^{\mathrm{TP}} h^{2}$, we can use Eq. (3.9) to determine the required re-heat temperature $T_{R}$. We plot our final results in the $m_{\tilde{a}}$ vs. $T_{R}$ plane, so that we may see whether a consistent cosmological scenario may be found for any of our Yukawa-unified solutions, and also whether a consistent cosmology helps to select out preferred values of the soft SUSY breaking parameters.

Our main results are shown in Fig. 11. Here, we generate Yukawa-unified solutions with $R \leq 1.05$ first with IsAJET 7.79 through MCMC scans for $m_{16}=5 \mathrm{TeV}$ (small-red 


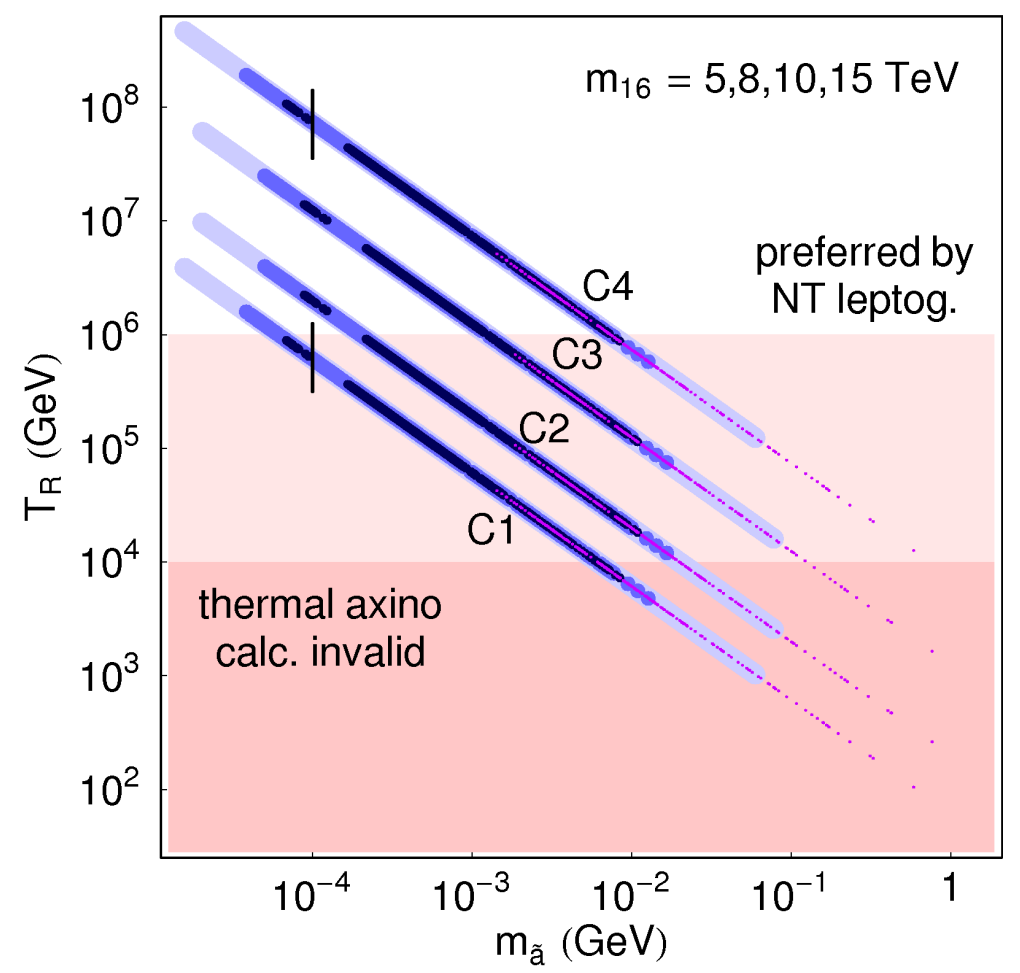

Figure 11: Plot of locus in the $m_{\tilde{a}} v s . T_{R}$ plane of four Yukawa-unified cases of mixed axion/axino dark matter, along with four different $m_{16}$ values. The thermally produced axino relic density calculation is only valid for $T_{R} \gtrsim 10^{4} \mathrm{GeV}$. Non-thermal leptogenesis prefers the region with $T_{R} \gtrsim$ $10^{6} \mathrm{GeV}$.

points), $8 \mathrm{TeV}$ (dark-blue larger points), $10 \mathrm{TeV}$ (medium-blue larger points) and $15 \mathrm{TeV}$ (very large light-blue points). The cases 1-4 are labelled as $\mathrm{C} 1-\mathrm{C} 4$. For the region below $T_{R} \sim 10^{4} \mathrm{GeV}$, the calculation of thermally produced axinos breaks down. Moreover, the value of $T_{R}$ is becoming comparable to the sparticle mass scale (i.e. the heavier sparticles will not be produced in thermal equilibrium in the early universe) so the calculation of $\Omega_{\tilde{\chi}_{1}^{0}} h^{2}$ would also break down. Values of $T_{R} \gtrsim 10^{6}$ are compatible with non-thermal leptogenesis via inflaton decay.

- We see from Fig. 11 that case $\mathrm{C} 1$ with $f_{a} / N=10^{11} \mathrm{GeV}$ and dominantly TP axino dark matter gives almost all solutions in the region with $T_{R}<10^{6} \mathrm{GeV}$. The solutions with $m_{16} \sim 5-8 \mathrm{TeV}$ especially have low values of $T_{R}$. The solutions with $m_{16} \sim 15$ TeV do have $T_{R} \gtrsim 10^{6} \mathrm{GeV}$, but these solutions also have $m_{\tilde{a}}<10^{-4} \mathrm{GeV}$, and so the dominant DM component from thermally produced axinos is likely warm DM. This scenario would thus be difficult to accept cosmologically, for any value of $m_{16}$.

- For case C2, we have $f_{a} / N=4 \times 10^{11} \mathrm{GeV}$ and dominant axion CDM. Here, the larger value of $f_{a} / N$ allows solutions with a similar value of $m_{\tilde{a}}$ as case C1, but with a higher value of $T_{R}$. The solutions with $m_{16}=10-15 \mathrm{TeV}$ do emerge with $T_{R} \gtrsim 10^{6}$ $\mathrm{GeV}$ - in the range for NT leptogenesis. Many of these solutions still have $m_{\tilde{a}}<10^{-4}$ 


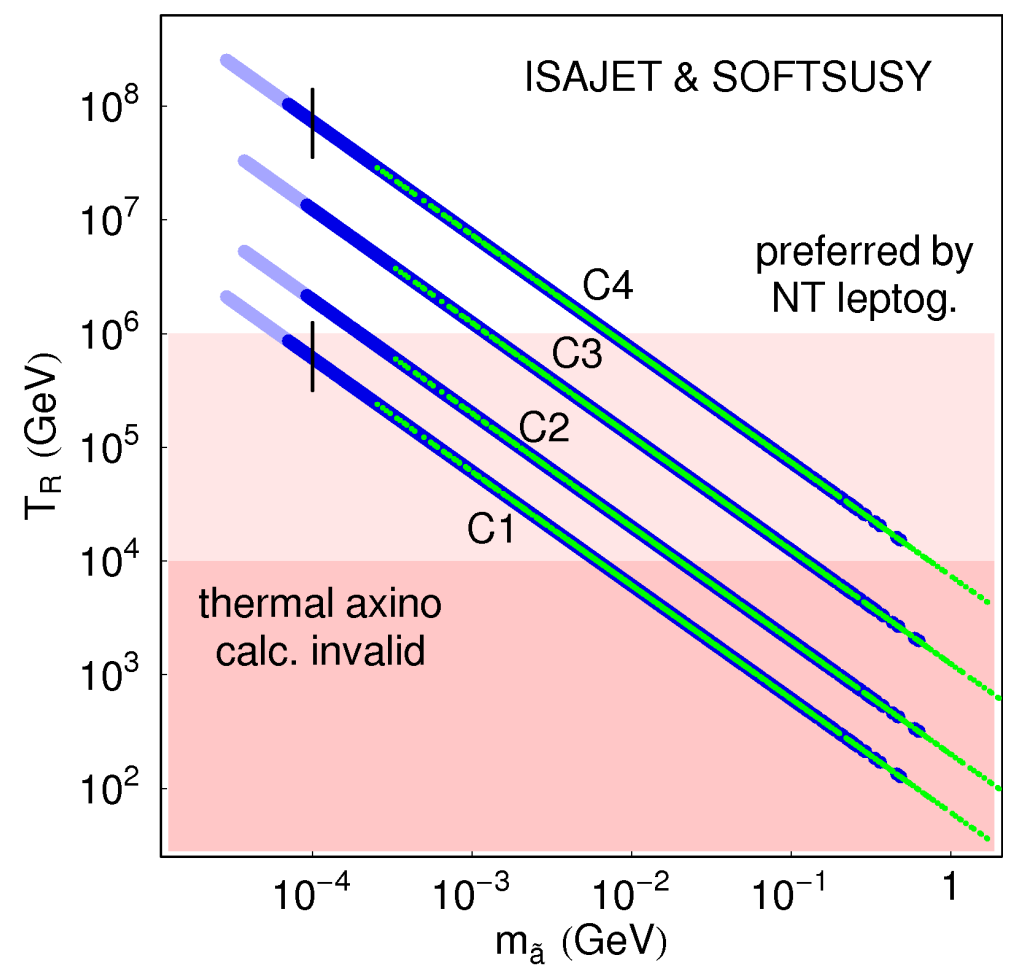

Figure 12: Plot of locus in the $m_{\tilde{a}} v s . T_{R}$ plane of four Yukawa-unified cases of mixed axion/axino dark matter for all $m_{16}$ values. We show results from IsAJET (blue) and SofTSUSY (green).

$\mathrm{GeV}$, so that the thermally produced axinos are warm. In this case, $m_{\tilde{a}}<10^{-4} \mathrm{GeV}$ is allowed, since instead the axions actually make up the CDM.

- In case C3, $f_{a} / N=10^{12} \mathrm{GeV}$ with dominant axion CDM. The larger $f_{a} / N$ gets, the larger are the calculated values of $T_{R}$. The thermal and non-thermal axinos both have small contributions to the relic density, so the entire band of solutions with $T_{R}>10^{6}$ $\mathrm{GeV}$ yields a consistent cosmology. This case requires an axino with $m_{\tilde{a}} \lesssim 10^{-3} \mathrm{GeV}$ for $T_{R}>10^{6} \mathrm{GeV}$.

- Finally, case C4 is constructed to have a large value of $f_{a} / N=10^{12} \mathrm{GeV}$, but with a tiny axion relic abundance due to accidental vacuum alignment. In this case, the thermally produced axinos comprise the CDM. Solutions are found with $T_{R}>10^{6}$ $\mathrm{GeV}$ for $m_{\tilde{a}} \lesssim 6 \times 10^{-3} \mathrm{GeV}$. However, in this case, the solutions with $m_{\tilde{a}} \lesssim 10^{-4}$ $\mathrm{GeV}$ would not be allowed, since they likely yield a dominant warm DM scenario, in contrast to requirements from large scale structure formation that the bulk of DM be cold.

In Fig. 12, we show Yukawa-unified solutions with $R<1.05$ for cases $\mathrm{C} 1-\mathrm{C} 4$ in the $m_{\tilde{a}}$ vs. $T_{R}$ plane from both IsAJET 7.79 and Softsusy 2.0.18 MCMC scans. ${ }^{3}$ IsAJET results are in blue, while SoftsusYresults are in green. Both sets of results line on the same line

\footnotetext{
${ }^{3}$ To be precise, the results for $m_{16}=5,8$, and $10 \mathrm{TeV}$ come from both IsAJET 7.79 and SofTSusy 2.0.18.
} 
for a given case. The IsAJET results reach to somewhat higher $T_{R}$ values than Softsusy. This is due in part because ISAJET can more easily generate Yukawa-unified models for

very high $m_{16} \gtrsim 10 \mathrm{TeV}$, and these models typically have larger $\Omega_{\tilde{\chi}_{1}^{0}} h^{2}$ values, and hence smaller values of $m_{\tilde{a}}$. Also, the SofTSUsy results tend to have lower $\mu$ values than ISAJET results, which also tends to lower the value of the neutralino relic density. Thus, case C1 does not lead to a consistent cosmology for either IsAJET or SofTSUsY. IsAJET can obtain cosmologies with $T_{R}>10^{6} \mathrm{GeV}$ for cases $\mathrm{C} 2-\mathrm{C} 4$, but SofTSUSy can generate cosmologies with $T_{R}>10^{6} \mathrm{GeV}$ only for case C3 (just barely) and case C4.

\section{Summary and conclusions}

One vestige of supersymmetric $S O(10)$ grand unified theories may be that the third generation $t-b-\tau$ Yukawa couplings unify, in addition to gauge couplings and matter multiplets. Assuming the MSSM is the low energy effective theory at energy scales $Q<M_{G U T}$, we are able to find parameter space solutions that yield a superparticle mass spectrum with Yukawa coupling unification good to $5 \%$ or better, using the IsAJET 7.79 and SofTSUSY 2.0.18 programs. The sparticle mass spectrum that qualitatively emerges is that first and second generation scalars lie in the multi-TeV regime, third generation scalars, $\mu$ and $m_{A}$ lie in the few $\mathrm{TeV}$ range, and gauginos lie in the sub-TeV range. The neutralino relic density turns out to be $10-10^{4}$ times the measured dark matter density, prompting the suggestion that in this case, the axino is a better LSP candidate, so that the dark matter of the universe would be composed of an axion/axino mix.

Yukawa-unified SUSY models should thus give rise to three components to the dark matter density: axion dark matter produced via vacuum mis-alignment at the QCD phase transition, non-thermally produced axinos from $\tilde{\chi}_{1}^{0} \rightarrow \tilde{a} \gamma$ decay (likely warm dark matter if $m_{\tilde{a}} \lesssim 1 \mathrm{GeV}$ ), and thermally produced axinos which are likely cold dark matter unless $m_{\tilde{a}} \lesssim 100 \mathrm{keV}$. We compute the abundance of all three components of dark matter in Yukawa-unified models for four different scenarios containing either dominant axion or dominant axino cold dark matter. The relative abundances depend on $\Omega_{\tilde{\chi}_{1}^{0}} h^{2}, m_{\tilde{\chi}_{1}^{0}}, m_{\tilde{a}}$, $f_{a} / N$ and $T_{R}$. It is found that for all solutions with $m_{16} \sim m_{3 / 2} \gtrsim 5 \mathrm{TeV}$, the value of $T_{R}$ needed is below limits calculated from BBN constraints, thus solving the gravitino BBN problem.

We also find that it is very difficult for models with PQ symmetry breaking scale $f_{a}$ lower than $\sim 2 \times 10^{11} \mathrm{GeV}$ (case $\mathrm{C} 1$ ) to generate dominantly cold dark matter and a sufficiently large $T_{R}$ to be consistent with at least non-thermal leptogenesis, for any allowed value of $m_{16}>5 \mathrm{TeV}$. However, if the PQ scale is large enough $\left(\gtrsim 4 \times 10^{11} \mathrm{GeV}\right)$, then we can generate a universe with axions as the dominant component of CDM (cases $\mathrm{C} 2$ and C3), and only a smaller component is composed of possibly warm axinos with $m_{\tilde{a}} \lesssim 10^{-3}$ $\mathrm{GeV}$. These solutions work out if $m_{16} \gtrsim 10 \mathrm{TeV}$, so that the higher side of the $m_{16} \sim 3-15$ $\mathrm{TeV}$ range is preferred. This is also in accord with results presented in Sec. 2.3, where it

Points with $m_{16}=15 \mathrm{TeV}$ come only from IsAJET 7.79, because Softsusy 2.0.18 does not give consistent EWSB for such high $m_{16}$. 
is found that $B F(b \rightarrow s \gamma)$ and $B F\left(B_{s} \rightarrow \mu^{+} \mu^{-}\right)$decay also prefer $m_{16} \gtrsim 10 \mathrm{TeV}$. Finally, we show a case $\mathrm{C} 4$ with accidentally small axion CDM component, but which gives cold thermally produced axino dark matter, as long as $m_{\tilde{a}} \gtrsim 100 \mathrm{keV}$. This scenario allows for solutions with $m_{16}$ as low as $5 \mathrm{TeV}$.

As far as tests go of the Yukawa-unified SUSY models with mixed axion/axino dark matter, it is clear from previous work that very large signal rates from gluino pair production and subsequent cascade decays should be visible at LHC relatively soon after start-up [66]. The $\tilde{\chi}_{1}^{0}$ produced in cascade decay events will still yield events with $E_{T}^{\text {miss }}$, since the $\tilde{\chi}_{1}^{0} \rightarrow \tilde{a} \gamma$ decay occurs far outside the detector. In cases C2 and C3, there is a good chance for direct detection of relic axion dark matter at experiments such as ADMX 67. However, direct and indirect searches for WIMP dark matter would likely turn up null results.

\section{A. Appendix: Yukawa-unified benchmark points from ISAJET 7.79}

Here, we present several updated benchmark points from IsAJET 7.79 with Yukawa-unified solutions. Points $\mathrm{A}, \mathrm{B}$ and $\mathrm{C}$ with $m_{16}=5,10$ and $15 \mathrm{TeV}$ respectively all require mixed axion/axino dark matter. Point $\mathrm{H}$ has $m_{16} \sim 3 \mathrm{TeV}$, and can accomodate neutralino dark matter since neutralino annihilation through the $h$ resonance leads to a neutralino dark matter abundance in near accord with WMAP5 measurements. ${ }^{4}$

\section{Acknowledgments}

This research was supported in part by the U.S. Department of Energy grant numbers DE-FG02-97ER41022. This work is also part of the French ANR project ToolsDMColl, BLAN07-2-194882. SK thanks the Kavli Institute for Theoretical Physics China (KITPC) for hospitality during the final stage of this work. SS acknowledges financial support by Turkish Atomic Energy Authority.

\section{References}

[1] S. Dimopoulos, S. Raby and F. Wilczek, Phys. Rev. D 24 (1981) 1681; U. Amaldi, W. de Boer and H. Furstenau, Phys. Lett. B 260 (1991) 447; J. Ellis, S. Kelley and D. V. Nanopoulos, Phys. Lett. B 260 (1991) 131; P. Langacker and Luo, Phys. Rev. D 44 (1991) 817 .

[2] H. Georgi and S. Glashow, Phys. Rev. Lett. 32 (1974) 438. H. Georgi, H. Quinn and S. Weinberg, Phys. Rev. Lett. 33 (1974) 451; A. Buras, J. Ellis, M. K. Gaillard and D. V. Nanopoulos, Nucl. Phys. B 135 (1978) 66.

[3] H. Georgi, in Proceedings of the American Institue of Physics, edited by C. Carlson (1974); H. Fritzsch and P. Minkowski, Ann. Phys. 93, 193 (1975); M. Gell-Mann, P. Ramond and R. Slansky, Rev. Mod. Phys. 50, 721 (1978). For recent reviews, see R. Mohapatra, hep-ph/9911272 (1999) and S. Raby, in Rept. Prog. Phys. 67 (2004) 755. For additional perspective, see G. Altarelli and F. Feruglio, hep-ph/0405048.

\footnotetext{
${ }^{4}$ Here, we anticipate a theory error bar on the relic density calculation arising from a variety of uncertainties to be of order $10-20 \%$.
} 


\begin{tabular}{|c|c|c|c|c|}
\hline parameter & Pt. A & Pt. B & Pt. C & Pt. H \\
\hline$m_{16}$ & 5000 & 10000 & 15000 & 2859.53 \\
\hline$m_{1 / 2}$ & 80.573 & 43.9442 & 15.2732 & 116.152 \\
\hline$A_{0}$ & -10046.7 & -19947.3 & -32495.5 & -5582.68 \\
\hline$m_{10}$ & 6273.26 & 12053.5 & 15098.8 & 3642.41 \\
\hline$M_{D}$ & 1655.92 & 3287.12 & 5367 & 943.32 \\
\hline $\tan \beta$ & 49.3631 & 50.398 & 51.7337 & 48.281 \\
\hline$f_{t}$ & 0.566 & 0.557 & 0.556 & 0.547 \\
\hline$f_{b}$ & 0.560 & 0.557 & 0.549 & 0.500 \\
\hline$f_{\tau}$ & 0.562 & 0.571 & 0.563 & 0.525 \\
\hline$R$ & 1.01 & 1.02 & 1.03 & 1.09 \\
\hline$\mu$ & 1101.2 & 3132.6 & 10365.8 & 440.4 \\
\hline$m_{\tilde{g}}$ & 363.3 & 351.2 & 368.8 & 406.4 \\
\hline$m_{\tilde{u}_{L}}$ & 4983.6 & 9972.1 & 14976.4 & 2855.7 \\
\hline$m_{\tilde{t}_{1}}$ & 833.7 & 2756.5 & 5422.5 & 318.6 \\
\hline$m_{\tilde{b}_{1}}$ & 1322.0 & 3377.1 & 6020.3 & 802.1 \\
\hline$m_{\tilde{e}_{L}}$ & 4968.1 & 9940.6 & 14904.2 & 2841.0 \\
\hline$m_{\tilde{\chi}_{1}^{ \pm}}$ & 109.2 & 116.4 & 136.2 & 115.7 \\
\hline$m_{\tilde{\chi}_{2}^{0}}$ & 108.9 & 113.8 & 135.5 & 115.1 \\
\hline$m_{\tilde{\chi}_{1}^{0}}$ & 49.8 & 49.2 & 54.4 & 56.6 \\
\hline$m_{A}$ & 939.0 & 1825.9 & 4714.9 & 884.3 \\
\hline$m_{h}$ & 124.1 & 127.8 & 128.4 & 115.0 \\
\hline$\Delta a_{\mu}$ & $0.4 \times 10^{-10}$ & $0.6 \times 10^{-11}$ & $0.5 \times 10^{-12}$ & $0.2 \times 10^{-9}$ \\
\hline$B F(b \rightarrow s \gamma)$ & $1.9 \times 10^{-4}$ & $3.0 \times 10^{-4}$ & $3.1 \times 10^{-4}$ & $1.3 \times 10^{-4}$ \\
\hline$B F\left(B_{s} \rightarrow \mu^{+} \mu^{-}\right)$ & $2.9 \times 10^{-8}$ & $8.1 \times 10^{-9}$ & $4.3 \times 10^{-9}$ & $3.6 \times 10^{-8}$ \\
\hline$\Omega h_{\tilde{\chi}_{1}^{0}}^{2}$ & 90.7 & 3881 & 522 & 0.13 \\
\hline
\end{tabular}

Table 2: Masses and parameters in GeV units for four cases studies using IsAJET 7.79 with $m_{t}=$ $172.6 \mathrm{GeV}$. We also list the $b \rightarrow s \gamma$ branching fraction, $\Delta a_{\mu}$ and $\Omega_{\tilde{\chi}_{1}^{0}} h^{2}$ for each case.

[4] M. Gell-Mann, P. Ramond and R. Slansky, in Supergravity, Proceedings of the Workshop, Stony Brook, NY 1979 (North-Holland, Amsterdam); T. Yanagida, KEK Report No. 79-18, 1979; R. Mohapatra and G. Senjanovic, Phys. Rev. Lett. 44 (1980) 912.

[5] B. Ananthanarayan, G. Lazarides and Q. Shafi, Phys. Rev. D 44 (1991) 1613 and Phys. Lett. B 300 (1993) 245; G. Anderson et al. Phys. Rev. D 47 (1993) 3702 and Phys. Rev. D 49 (1994) 3660; V. Barger, M. Berger and P. Ohmann, Phys. Rev. D 49 (1994) 4908; M. Carena, M. Olechowski, S. Pokorski and C. Wagner, Ref. [7]; B. Ananthanarayan, Q. Shafi and X. Wang, Phys. Rev. D 50 (1994) 5980; R. Rattazzi and U. Sarid, Phys. Rev. D 53 (1996) 1553; T. Blazek, M. Carena, S. Raby and C. Wagner, Phys. Rev. D 56 (1997) 6919; T. Blazek and S. Raby, Phys. Lett. B 392 (1997) 371; T. Blazek and S. Raby, Phys. Rev. D 59 (1999) 095002; T. Blazek, S. Raby and K. Tobe, Phys. Rev. D 60 (1999) 113001 and Phys. Rev. D 62 (2000) 055001; H. Baer, M. Diaz, J. Ferrandis and X. Tata, Phys. Rev. D 61 (2000) 111701; H. Baer, M. Brhlik, M. Diaz, J. Ferrandis, P. Mercadante, P. Quintana and X. Tata, Phys. Rev. D 63 (2001) 015007; S. Profumo, Phys. Rev. D 68 (2003) 015006 ; 
C. Pallis, Nucl. Phys. B 678 (2004) 398; M. Gomez, G. Lazarides and C. Pallis, Phys. Rev. D 61 (2000) 123512, Nucl. Phys. B 638 (2002) 165 and Phys. Rev. D 67 (2003) 097701; U. Chattopadhyay, A. Corsetti and P. Nath, Phys. Rev. D 66 (2002) 035003; M. Gomez, T. Ibrahim, P. Nath and S. Skadhauge, Phys. Rev. D 72 (2005) 095008; K. Tobe and J. D. Wells, Nucl. Phys. B 663 (2003) 123.

[6] Y. Kawamura, Prog. Theor. Phys. 105 (2001) 999; G. Altarelli and F. Feruglio, Phys. Lett. B 511 (2001) 257; L. Hall and Y. Nomura, Phys. Rev. D 64 (2001) 055003; A. Hebecker and J. March-Russell, Nucl. Phys. B 613 (2001) 3; A. Kobakhidze, Phys. Lett. B 514 (2001) 131.

[7] R. Hempfling, Phys. Rev. D 49 (1994) 6168; L. J. Hall, R. Rattazzi and U. Sarid, Phys. Rev. D 50 (1994) 7048; M. Carena et al., Nucl. Phys. B 426 (1994) 269.

[8] T. Blazek, R. Dermisek and S. Raby, Phys. Rev. D 65 (2002) 115004.

[9] H. Baer and J. Ferrandis, Phys. Rev. Lett. 87 (2001) 211803.

[10] D. Auto, H. Baer, C. Balazs, A. Belyaev, J. Ferrandis and X. Tata, J. High Energy Phys. $0306(2003) 023$.

[11] H. Baer, S. Kraml, S. Sekmen and H. Summy, J. High Energy Phys. 0803 (2008) 056.

[12] F. Paige, S. Protopopescu, H. Baer and X. Tata, hep-ph/0312045; http://www.hep.fsu.edu/ isajet/

[13] T. Blazek, R. Dermisek and S. Raby, Phys. Rev. Lett. 88 (2002) 111804.

[14] R. Dermisek, S. Raby, L. Roszkowski and R. Ruiz de Austri, J. High Energy Phys. 0304 (2003) 037 and J. High Energy Phys. 0509 (2005) 029.

[15] J. Feng, C. Kolda and N. Polonsky, Nucl. Phys. B 546 (1999) 3; J. Bagger, J. Feng and N. Polonsky, Nucl. Phys. B 563 (1999) 3; J. Bagger, J. Feng, N. Polonsky and R. Zhang, Phys. Lett. B 473 (2000) 264; H. Baer,P. Mercadante and X. Tata, Phys. Lett. B 475 (2000) 289; H. Baer, C. Balazs, M. Brhlik, P. Mercadante, X. Tata and Y. Wang, Phys. Rev. D 64 (2001) 015002; see also H. Baer, M. Diaz, P. Quintana and X. Tata, J. High Energy Phys. 0004 (2000) 016.

[16] D. Auto, H. Baer and A. Belyaev and T. Krupovnickas, J. High Energy Phys. 0410 (2004) 066 .

[17] D. N. Spergel et al. (WMAP Collaboration), Astrophys. J. Supp., 170 (2007) 377.

[18] R. Peccei and H. Quinn, Phys. Rev. Lett. 38 (1977) 1440 and Phys. Rev. D 16 (1977) 1791.

[19] S. Weinberg, Phys. Rev. Lett. 40 (1978) 223; F. Wilczek, Phys. Rev. Lett. 40 (1978) 279.

[20] For recent reviews on axion physics, see J. E. Kim and G. Carosi, arXiv:0807.3125 (2008); P. Sikivie, hep-ph/0509198; M. Turner, Phys. Rept. 197 (1990) 67.

[21] H. P. Nilles and S. Raby, Nucl. Phys. B 198 (1982) 102.

[22] E. J. Chun, J. E. Kim and H. P. Nilles, Phys. Lett. B 287 (1992) 123.

[23] L. Covi, J. E. Kim and L. Roszkowski, Phys. Rev. Lett. 82 (1999) 4180; L. Covi, H. B. Kim, J. E. Kim and L. Roszkowski, J. High Energy Phys. 0105 (2001) 033.

[24] K. Rajagopal, M. Turner and F. Wilczek, Nucl. Phys. B 358 (1991) 447.

[25] For a recent review of axion/axino dark matter, see F. Steffen, arXiv:0811.3347 (2008). 
[26] K. Jedamzik, M. LeMoine and G. Moultaka, JCAP0607 (2006) 010.

[27] A. Brandenburg and F. Steffen, JCAP0408 (2004) 008.

[28] A. Boyarsky, J. Lesgourgues, O. Ruchayskiy and M. Viel, arXiv:0812.0010 [astro-ph].

[29] K. Kohri, T. Moroi and A. Yotsuyanagi, Phys. Rev. D 73 (2006) 123511; for an update, see M. Kawasaki, K. Kohri, T. Moroi and A. Yotsuyanagi, arXiv:0804.3745 (2008).

[30] W. Buchmuller, P. Di Bari and M. Plumacher, Annal. Phys. 315 (2005) 305.

[31] G. Lazarides and Q. Shafi, Phys. Lett. B 258 (1991) 305; K. Kumekawa, T. Moroi and T. Yanagida, Prog. Theor. Phys. 92 (1994) 437; T. Asaka, K. Hamaguchi, M. Kawasaki and T. Yanagida, Phys. Lett. B 464 (1999) 12.

[32] H. Baer and H. Summy, Phys. Lett. B 666 (2008) 5.

[33] The Tevatron Electroweak Working group (CDF and D0 Collaborations), arXiv:0803.1683.

[34] D. Castano, Piard and P. Ramond, Phys. Rev. D 49 (1994) 4882, A. Dedes, A. Lahanas and K. Tamvakis, Phys. Rev. D 53 (1996) 3793.

[35] A. Box and X. Tata, Phys. Rev. D 77 (2008) 055007 and arXiv:0810.5765 (2008).

[36] D. Pierce, J. Bagger, K. Matchev and R. Zhang, Nucl. Phys. B 491 (1997) 3.

[37] S. P. Martin and M. Vaughn, Phys. Rev. D 50 (1994) 2282.

[38] H. Baer, J. Ferrandis, S. Kraml and W. Porod, Phys. Rev. D 73 (2006) 015010.

[39] B.C. Allanach, S. Kraml and W. Porod, J. High Energy Phys. 03 (2003) 016; G. Belanger, S. Kraml and A. Pukhov, Phys. Rev. D 72 (2005) 015003; S. Kraml and S. Sekmen in: M.M. Nojiri et al., Physics at TeV Colliders 2007, BSM working group report, arXiv:0802.3672 [hep-ph].

[40] B. Allanach, Comput. Phys. Commun. 143 (2002) 305.

[41] G. Belanger, F. Boudjema, A. Pukhov and A. Semenov, Comput. Phys. Commun. 149 (2002) 103, Comput. Phys. Commun. 174 (2006) 577 and Comput. Phys. Commun. 176 (2007) 367.

[42] H. Baer, C. Balazs, A. Belyaev, J. K. Mizukoshi and X. Tata, J. High Energy Phys. 0207 (2002) 050.

[43] H. Baer and M. Brhlik, Phys. Rev. D 55 (1997) 3201.

[44] H. Anlauf, Nucl. Phys. B 430 (1994) 245.

[45] C. Greub, T. Hurth and D. Wyler, Phys. Lett. B 380 (1996) 385 and Phys. Rev. D 54 (1996) 3350.

[46] H. Baer, M. Brhlik, D. Castano and X. Tata, Phys. Rev. D 58 (1998) 015007.

[47] M. Misiak et al., Phys. Rev. Lett. 98 (2007) 022002.

[48] E. Barberio et al. (Heavy Flavor Averaging Group), hep-ex/0603003.

[49] M. Albrecht, W. Altmannshofer, A. Buras, D. Guadagnoli and D. Straub, J. High Energy Phys. 0710 (2007) 055; W. Altmannshofer, D. Guadagnoli, S. Raby and D. Straub, arXiv:0801.4363 (2008); D. Guadagnoli, arXiv:0810.0450 (2008).

[50] J. K. Mizukoshi, X. Tata and Y. Wang, Phys. Rev. D 66 (2002) 115003. 
[51] T. Aaltonen et al. (CDF Collaboration), Phys. Rev. Lett. 100 (2008) 101802.

[52] L. F. Abbott and P. Sikivie, Phys. Lett. B 120 (1983) 133; J. Preskill, M. Wise and F. Wilczek, Phys. Lett. B 120 (1983) 127; M. Dine and W. Fischler, Phys. Lett. B 120 (1983) 137; M. Turner, Phys. Rev. D 33 (1986) 889.

[53] H. Baer and X. Tata, Weak Scale Supersymmetry: From Superfields to Scattering Events, (Cambridge University Press, 2006).

[54] J. E. Kim, Phys. Rev. Lett. 43 (1979) 103; M. A. Shifman, A. Vainstein and V. I. Zakharov, Nucl. Phys. B 166 (1980) 493.

[55] M. Dine, W. Fischler and M. Srednicki, Phys. Lett. B 104 (1981) 199; A. P. Zhitnitskii, Sov. J. Nucl. 31 (1980) 260.

[56] E. Braaten and R. Pisarski, Nucl. Phys. B 337 (1990) 569; E. Braaten and T. C. Yuan, Phys. Rev. Lett. 66 (1991) 2183.

[57] E. Cremmer, S. Ferrara, L. Girardello and A. van Proeyen, Nucl. Phys. B 212 (1983) 413.

[58] H. P. Nilles, Phys. Rept. 110 (1984)1.

[59] M. Bolz, A. Brandenburg and W. Buckmuller, Nucl. Phys. B 606 (2001) 518; J. Pradler and F. Steffen, Phys. Rev. D 75 (2007) 023509.

[60] S. Weinberg, Phys. Rev. Lett. 48 (1982) 1303; R. H. Cyburt, J. Ellis, B. D. Fields and K. A. Olive, Phys. Rev. D 67 (2003) 103521: K. Jedamzik, Phys. Rev. D 70 (2004) 063524; M. Kawasaki, K. Kohri and T. Moroi, Phys. Lett. B 625 (2005) 7 and Phys. Rev. D 71 (2005) 083502 .

[61] M. Fukugita and T. Yanagida, Phys. Lett. B 174 (1986) 45; M. Luty, Phys. Rev. D 45 (1992) 455; M. Luty, Phys. Rev. D 45 (1992) 455; W. Buchmüller and M. Plumacher, Phys. Lett. B 389 (1996) 73 and Int. J. Mod. Phys. A 15 (2000) 5047; R. Barbieri, P. Creminelli, A. Strumia and N. Tetradis, Nucl. Phys. B 575 (2000) 61; G. F. Giudice, A. Notari, M. Raidal, A. Riotto and A. Strumia, hep-ph/0310123; for a recent review, see W. Buchmüller, R. Peccei and T. Yanagida, Ann. Rev. Nucl. Part. Sci. 55 (2005) 311.

[62] G. Lazarides and Q. Shafi, Phys. Lett. B 258 (1991) 305; K. Kumekawa, T. Moroi and T. Yanagida, Prog. Theor. Phys. 92 (1994) 437; T. Asaka, K. Hamaguchi, M. Kawasaki and T. Yanagida, Phys. Lett. B 464 (1999) 12.

[63] K. Hamaguchi, H. Murayama and T. Yanagida, Phys. Rev. D 65 (2002) 043512.

[64] W. Buchmüller, P. Di Bari and M. Plumacher, Nucl. Phys. B 643 (2002) 367 and Erratum-ibid,B793 (2008) 362; Annals Phys. 315 (2005) 305 and New J. Phys. 6 (2004) 105.

[65] M. Ibe, T. Moroi and T. Yanagida, Phys. Lett. B 620 (2005) 9.

[66] H. Baer, S. Kraml, S. Sekmen and H. Summy, J. High Energy Phys. 0810 (2008) 079.

[67] L. Duffy et al., Phys. Rev. Lett. 95 (2005) 091304 and Phys. Rev. D 74 (2006) 012006; for a review, see S. Asztalos, L. Rosenberg, K. van Bibber, P. Sikivie and K. Zioutas, Ann. Rev. Nucl. Part. Sci. 56 (2006) 293. 